TRANSACTIONS OF THE

AMERICAN MATHEMATICAL SOCIETY

Volume 363, Number 7, July 2011, Pages 3639-3663

S 0002-9947(2011)05247-5

Article electronically published on February 3, 2011

\title{
ASYMPTOTIC BEHAVIOR OF STOCHASTIC WAVE EQUATIONS WITH CRITICAL EXPONENTS ON $\mathbb{R}^{3}$
}

\author{
BIXIANG WANG
}

\begin{abstract}
The existence of a random attractor in $H^{1}\left(\mathbb{R}^{3}\right) \times L^{2}\left(\mathbb{R}^{3}\right)$ is proved for the damped semilinear stochastic wave equation defined on the entire space $\mathbb{R}^{3}$. The nonlinearity is allowed to have a cubic growth rate which is referred to as the critical exponent. The uniform pullback estimates on the tails of solutions for large space variables are established. The pullback asymptotic compactness of the random dynamical system is proved by using these tail estimates and the energy equation method.
\end{abstract}

\section{INTRODUCTION}

This paper deals with the existence of a random attractor for the stochastic wave equation defined on $\mathbb{R}^{3}$ :

$$
u_{t t}+\alpha u_{t}-\Delta u+\lambda u+f(x, u)=g(x)+h(x) \frac{d w}{d t},
$$

with the initial conditions

$$
u(x, \tau)=u_{0}(x), \quad u_{t}(x, \tau)=u_{1}(x),
$$

where $x \in \mathbb{R}^{3}, t>\tau$ with $\tau \in \mathbb{R}, \alpha$ and $\lambda$ are positive numbers, $g$ and $h$ are given in $L^{2}\left(\mathbb{R}^{3}\right)$ and $H^{1}\left(\mathbb{R}^{3}\right)$, respectively, $f$ is a nonlinear function with cubic growth rate (called the critical exponent), and $w$ is an independent two-sided real-valued Wiener process on a probability space.

The global attractors of the deterministic wave equation (i.e. $h=0$ ) have been studied extensively in the literature; see, e.g., 3, 4, 20, 30, 34 and the references therein. Particularly, the existence of these attractors was proved in 2, 3, 4, 11, 18, 22, 32, 33. for the deterministic equation defined in bounded domains with critical exponents, and in [16, 17, 27, 28, 29] for the equation defined on unbounded domains with critical or supercritical exponents. In this paper, we will investigate the asymptotic behavior of the stochastic wave equation (1.1) with critical exponents defined on the entire space $\mathbb{R}^{3}$.

The interesting features of problem (1.1)-(1.2) lie in: (i) The equation is stochastic. In this case, problem (1.1)-(1.2) determines a random dynamical system instead of a deterministic semigroup; (ii) The nonlinearity $f$ is critical. The difficulty caused by the noncompactness of embedding $H^{1} \hookrightarrow L^{6}$ must be overcome

Received by the editors May 1, 2009 and, in revised form, November 2, 2009.

2000 Mathematics Subject Classification. Primary 37L55; Secondary 60H15, 35B40.

Key words and phrases. Random attractor, asymptotic compactness, wave equation.

The author was supported in part by NSF grant DMS-0703521.

(C)2011 American Mathematical Society 
in order to deal with the asymptotic compactness of solutions with such a critical nonlinearity; (iii) The domain $\mathbb{R}^{3}$ of problem (1.1)-(1.2) is unbounded. In this case, the embeddings $H^{1}\left(\mathbb{R}^{3}\right) \hookrightarrow L^{p}\left(\mathbb{R}^{3}\right)$ are not compact even for $p<6$. This is essentially different from the case of bounded domains.

To study the long term behavior of solutions of stochastic differential equations, the concept of random attractor should be used instead of global attractor, which was introduced in [14, 19] for random dynamical systems. Since the nonlinearity $f$ of equation (1.1) has a critical growth rate, the mapping $f$ from $H^{1}(Q)$ to $L^{2}(Q)$ is continuous, but not compact, even for a bounded domain $Q$ in $\mathbb{R}^{3}$. To circumvent the difficulty and prove the asymptotic compactness of the deterministic wave equation on a bounded domain $Q$, an energy equation approach was developed by Ball in [4. This method is quite effective for a variety of applications; see, e.g., [5, 24, 25, 26, 40. Notice that the compactness of the embeddings $H^{1}(Q) \hookrightarrow L^{p}(Q)$ with $p<6$ was crucial and frequently used in [4] when $Q$ is bounded. In our case, the domain $\mathbb{R}^{3}$ is unbounded, and hence the embeddings $H^{1}\left(\mathbb{R}^{3}\right) \hookrightarrow L^{p}\left(\mathbb{R}^{3}\right)$ are not compact for any $p$. This means that Ball's method [4 alone is not sufficient for proving the asymptotic compactness of the equation on $\mathbb{R}^{3}$. We must overcome the difficulty caused by the noncompactness of the embeddings $H^{1}\left(\mathbb{R}^{3}\right) \hookrightarrow L^{p}\left(\mathbb{R}^{3}\right)$ for $p<6$. In this paper, we will solve the problem by using uniform estimates on the tails of solutions. We will first show that the solutions of problem (1.1)-(1.2) uniformly approach zero, in a sense, as $x$ and $t$ go to infinity, and then apply these estimates and the energy equation method 4 to prove the asymptotic compactness of the stochastic wave equations on $\mathbb{R}^{3}$.

The random attractors of stochastic partial differential equations defined in bounded domains have been extensively investigated by many authors in 9, 10, 12, 13, 14, 19, 23, 41] and the references therein. However, when the domains are unbounded, the existence of such attractors is not well understood. Recently, the existence and upper semicontinuity of random attractors for some equations on unbounded domains have been established in [7, 36, 37, 38] and [39, respectively. The asymptotic compactness of the stochastic Navier-Stokes equations on unbounded domains was proved in 8 . In this paper, we will investigate the existence of a random attractor for the stochastic wave equation with critical nonlinearity on $\mathbb{R}^{3}$.

This paper is organized as follows. In the next section, we recall the random attractors theory for random dynamical systems. In Section 3, we define a continuous random dynamical system for problem (1.1)-(1.2). The uniform estimates of solutions are contained in Section 4, which include uniform estimates on the tails of solutions. In Section 5, we prove the pullback asymptotic compactness and the existence of random attractors for the stochastic wave equation on $\mathbb{R}^{3}$.

In the sequel, we adopt the following notation. We denote by $\|\cdot\|$ and $(\cdot, \cdot)$ the norm and the inner product of $L^{2}\left(\mathbb{R}^{3}\right)$, respectively. The norm of a given Banach space $X$ is written as $\|\cdot\|_{X}$. We also use $\|\cdot\|_{p}$ to denote the norm of $L^{p}\left(\mathbb{R}^{3}\right)$. The letters $c$ and $c_{i}(i=1,2, \ldots)$ are generic positive constants which may change their values from line to line or even in the same line.

\section{Preliminaries}

In this section, we recall some basic concepts related to random attractors for stochastic dynamical systems. The reader is referred to [1, 6, 13, 19] for more details. 
Let $\left(X,\|\cdot\|_{X}\right)$ be a separable Hilbert space with Borel $\sigma$-algebra $\mathcal{B}(X)$, and let $(\Omega, \mathcal{F}, P)$ be a probability space.

Definition 2.1. $\left(\Omega, \mathcal{F}, P,\left(\theta_{t}\right)_{t \in \mathbb{R}}\right)$ is called a metric dynamical system if $\theta: \mathbb{R} \times$ $\Omega \rightarrow \Omega$ is $(\mathcal{B}(\mathbb{R}) \times \mathcal{F}, \mathcal{F})$-measurable, $\theta_{0}$ is the identity on $\Omega, \theta_{s+t}=\theta_{t} \circ \theta_{s}$ for all $s, t \in \mathbb{R}$ and $\theta_{t} P=P$ for all $t \in \mathbb{R}$.

Definition 2.2. A continuous random dynamical system (RDS) on $X$ over a metric dynamical system $\left(\Omega, \mathcal{F}, P,\left(\theta_{t}\right)_{t \in \mathbb{R}}\right)$ is a mapping

$$
\Phi: \mathbb{R}^{+} \times \Omega \times X \rightarrow X, \quad(t, \omega, x) \mapsto \Phi(t, \omega, x),
$$

which is $\left(\mathcal{B}\left(\mathbb{R}^{+}\right) \times \mathcal{F} \times \mathcal{B}(X), \mathcal{B}(X)\right)$-measurable and satisfies, for $P$-a.e. $\omega \in \Omega$,

(i) $\Phi(0, \omega, \cdot)$ is the identity on $X$;

(ii) $\Phi(t+s, \omega, \cdot)=\Phi\left(t, \theta_{s} \omega, \cdot\right) \circ \Phi(s, \omega, \cdot)$ for all $t, s \in \mathbb{R}^{+}$;

(iii) $\Phi(t, \omega, \cdot): X \rightarrow X$ is continuous for all $t \in \mathbb{R}^{+}$.

Hereafter, we always assume that $\Phi$ is a continuous RDS on $X$ over $(\Omega, \mathcal{F}, P$, $\left.\left(\theta_{t}\right)_{t \in \mathbb{R}}\right)$.

Definition 2.3. A random bounded set $\{B(\omega)\}_{\omega \in \Omega}$ of $X$ is called tempered with respect to $\left(\theta_{t}\right)_{t \in \mathbb{R}}$ if for $P$-a.e. $\omega \in \Omega$,

$$
\lim _{t \rightarrow \infty} e^{-\beta t} d\left(B\left(\theta_{-t} \omega\right)\right)=0 \quad \text { for all } \beta>0,
$$

where $d(B)=\sup _{x \in B}\|x\|_{X}$.

Definition 2.4. A random function $r(\omega)$ is called tempered with respect to $\left(\theta_{t}\right)_{t \in \mathbb{R}}$ if for $P$-a.e. $\omega \in \Omega$,

$$
\lim _{t \rightarrow \infty} e^{-\beta t} r\left(\theta_{-t} \omega\right)=0 \quad \text { for all } \beta>0 .
$$

Definition 2.5. Let $\mathcal{D}$ be a collection of random subsets of $X$. Then $\mathcal{D}$ is called inclusion-closed if $D=\{D(\omega)\}_{\omega \in \Omega} \in \mathcal{D}$ and $\tilde{D}=\{\tilde{D}(\omega) \subseteq X: \omega \in \Omega\}$ with $\tilde{D}(\omega) \subseteq D(\omega)$ for all $\omega \in \Omega$ imply that $\tilde{D} \in \mathcal{D}$.

Definition 2.6. Let $\mathcal{D}$ be a collection of random subsets of $X$ and $\{K(\omega)\}_{\omega \in \Omega} \in \mathcal{D}$. Then $\{K(\omega)\}_{\omega \in \Omega}$ is called an absorbing set of $\Phi$ in $\mathcal{D}$ if for every $B \in \mathcal{D}$ and $P$-a.e. $\omega \in \Omega$, there exists $t_{B}(\omega)>0$ such that

$$
\Phi\left(t, \theta_{-t} \omega, B\left(\theta_{-t} \omega\right)\right) \subseteq K(\omega) \text { for all } t \geq t_{B}(\omega) .
$$

Definition 2.7. Let $\mathcal{D}$ be a collection of random subsets of $X$. Then $\Phi$ is said to be $\mathcal{D}$-pullback asymptotically compact in $X$ if for $P$-a.e. $\omega \in \Omega,\left\{\Phi\left(t_{n}, \theta_{-t_{n}} \omega, x_{n}\right)\right\}_{n=1}^{\infty}$ has a convergent subsequence in $X$ whenever $t_{n} \rightarrow \infty$, and $x_{n} \in B\left(\theta_{-t_{n}} \omega\right)$ with $\{B(\omega)\}_{\omega \in \Omega} \in \mathcal{D}$.

Definition 2.8. Let $\mathcal{D}$ be a collection of random subsets of $X$ and $\{\mathcal{A}(\omega)\}_{\omega \in \Omega} \in \mathcal{D}$. Then $\{\mathcal{A}(\omega)\}_{\omega \in \Omega}$ is called a $\mathcal{D}$-random attractor (or $\mathcal{D}$-pullback attractor) for $\Phi$ if the following conditions are satisfied, for $P$-a.e. $\omega \in \Omega$ :

(i) $\mathcal{A}(\omega)$ is compact, and $\omega \mapsto d(x, \mathcal{A}(\omega))$ is measurable for every $x \in X$;

(ii) $\{\mathcal{A}(\omega)\}_{\omega \in \Omega}$ is invariant, that is,

$$
\Phi(t, \omega, \mathcal{A}(\omega))=\mathcal{A}\left(\theta_{t} \omega\right), \quad \forall t \geq 0
$$

(iii) $\{\mathcal{A}(\omega)\}_{\omega \in \Omega}$ attracts every set in $\mathcal{D}$, that is, for every $B=\{B(\omega)\}_{\omega \in \Omega} \in \mathcal{D}$,

$$
\lim _{t \rightarrow \infty} d\left(\Phi\left(t, \theta_{-t} \omega, B\left(\theta_{-t} \omega\right)\right), \mathcal{A}(\omega)\right)=0
$$


where $d$ is the Hausdorff semi-metric given by $d(Y, Z)=\sup _{y \in Y} \inf _{z \in Z}\|y-z\|_{X}$ for any $Y \subseteq X$ and $Z \subseteq X$.

The following existence result on a random attractor for a continuous RDS can be found in [6, 19.

Proposition 2.9. Let $\mathcal{D}$ be an inclusion-closed collection of random subsets of $X$ and $\Phi$ a continuous $R D S$ on $X$ over $\left(\Omega, \mathcal{F}, P,\left(\theta_{t}\right)_{t \in \mathbb{R}}\right)$. Suppose that $\{K(\omega)\}_{\omega \in K}$ is a closed absorbing set of $\Phi$ in $\mathcal{D}$ and $\Phi$ is $\mathcal{D}$-pullback asymptotically compact in $X$. Then $\Phi$ has a unique $\mathcal{D}$-random attractor $\{\mathcal{A}(\omega)\}_{\omega \in \Omega}$ which is given by

$$
\mathcal{A}(\omega)=\bigcap_{\tau \geq 0} \overline{\bigcup_{t \geq \tau} \Phi\left(t, \theta_{-t} \omega, K\left(\theta_{-t} \omega\right)\right)} .
$$

In this paper, we will denote by $\mathcal{D}$ the collection of all tempered random sets of $H^{1}\left(\mathbb{R}^{3}\right) \times L^{2}\left(\mathbb{R}^{3}\right)$ and prove that problem (1.1)-(1.2) has a $\mathcal{D}$-random attractor.

\section{RANDOM DYNAMICAL SYSTEMS}

In this section, we define a continuous random dynamical system for problem (1.1)-(1.2). Denote by $z=u_{t}+\delta u$, where $\delta$ is a small positive number to be determined later. Substituting $u_{t}=z-\delta u$ into (1.1) we find that

$$
\begin{gathered}
\frac{d u}{d t}+\delta u=z \\
\frac{d z}{d t}+(\alpha-\delta) z+\left(\lambda+\delta^{2}-\alpha \delta\right) u-\Delta u+f(x, u)=g(x)+h(x) \frac{d w}{d t},
\end{gathered}
$$

with the initial conditions

$$
u(x, \tau)=u_{0}(x), \quad z(x, \tau)=z_{0}(x),
$$

where $z_{0}(x)=u_{1}(x)+\delta u_{0}(x), x \in \mathbb{R}^{3}, t>\tau$ with $\tau \in \mathbb{R}, \alpha$ and $\lambda$ are positive numbers, $g \in L^{2}\left(\mathbb{R}^{3}\right)$ and $h \in H^{1}\left(\mathbb{R}^{3}\right)$ are given, and $w$ is an independent two-sided real-valued Wiener process on a complete probability space $(\Omega, \mathcal{F}, P)$ with path $\omega(\cdot)$ in $C(\mathbb{R}, \mathbb{R})$ satisfying $\omega(0)=0$. The reader is referred to [15] for more details on the theory of Wiener processes. Let $\left(\theta_{t}\right)_{t \in \mathbb{R}}$ be a family of measure-preserving shift operators given by

$$
\theta_{t} \omega(\cdot)=\omega(\cdot+t)-\omega(t), \quad \forall \omega \in \Omega \text { and } t \in \mathbb{R} .
$$

Then $\left(\Omega, \mathcal{F}, P,\left(\theta_{t}\right)_{t \in \mathbb{R}}\right)$ forms a metric dynamical system. Let $F(x, u)=\int_{0}^{u} f(x, s) d s$ for $x \in \mathbb{R}^{3}$ and $u \in \mathbb{R}$. We assume the following conditions on the the nonlinearity $f$, for every $x \in \mathbb{R}^{3}$ and $u \in \mathbb{R}$ :

$$
\begin{gathered}
|f(x, u)| \leq c_{1}|u|^{\gamma}+\phi_{1}(x), \quad \phi_{1} \in L^{2}\left(\mathbb{R}^{3}\right), \\
f(x, u) u-c_{2} F(x, u) \geq \phi_{2}(x), \quad \phi_{2} \in L^{1}\left(\mathbb{R}^{3}\right), \\
F(x, u) \geq c_{3}|u|^{\gamma+1}-\phi_{3}, \quad \phi_{3} \in L^{1}\left(\mathbb{R}^{3}\right), \\
\left|f_{u}(x, u)\right| \leq c_{4}|u|^{\gamma-1}+\phi_{4}, \quad \phi_{4} \in H^{1}\left(\mathbb{R}^{3}\right),
\end{gathered}
$$

where $1 \leq \gamma \leq 3$. As a special case, $\gamma=3$ is referred to as the critical exponent. 
Notice that (3.4) and (3.5) imply

$$
F(x, u) \leq c\left(|u|^{2}+|u|^{\gamma+1}+\phi_{1}^{2}+\phi_{2}\right),
$$

which is useful when deriving uniform estimates of solutions.

A pair $(u, z)$ is called a solution of problem (3.1)-(3.3) on the interval $[\tau, \tau+T]$ if $(u, z) \in L^{2}\left(\Omega ; C\left([\tau, \tau+T] ; H^{1}\left(\mathbb{R}^{3}\right) \times L^{2}\left(\mathbb{R}^{3}\right)\right)\right)$ such that, for almost every $\omega \in \Omega$, (3.3) is satisfied and (3.1)-(3.2) hold in the sense of distributions over $\mathbb{R}^{3} \times(\tau, \tau+T)$. In order to study the dynamical behavior of problem (3.1)-(3.3), we need to convert the stochastic system into a deterministic one with a random parameter. To this end, we set $v(t, \tau, \omega)=z(t, \tau, \omega)-h \omega(t)$. Then it follows from (3.1)-3.3 that

$$
\begin{gathered}
\frac{d u}{d t}+\delta u-v=h \omega(t) \\
\frac{d v}{d t}+(\alpha-\delta) v+\left(\lambda+\delta^{2}-\alpha \delta\right) u-\Delta u+f(x, u)=g+(\delta-\alpha) h \omega(t),
\end{gathered}
$$

with the initial conditions

$$
u(x, \tau)=u_{0}(x), \quad v(x, \tau)=v_{0}(x),
$$

where $v_{0}(x)=z_{0}(x)-h \omega(\tau)$.

The existence, uniqueness and regularity of solutions for deterministic wave equations have been studied by many authors; see, e.g., 21 for the case of bounded domains and [27, 31] for unbounded domains. Similarly, it can be proved that problem (3.9)-(3.11) with (3.4)-(3.7) is well-posed in $H^{1}\left(\mathbb{R}^{3}\right) \times L^{2}\left(\mathbb{R}^{3}\right)$; that is, for $P$-a.e. $\omega \in \Omega$, for every $\tau \in \mathbb{R}$ and $\left(u_{0}, v_{0}\right) \in H^{1}\left(\mathbb{R}^{3}\right) \times L^{2}\left(\mathbb{R}^{3}\right)$, problem (3.9)(3.11) has a unique solution $(u(\cdot, \tau, \omega), v(\cdot, \tau, \omega)) \in C\left([\tau, \infty), H^{1}\left(\mathbb{R}^{3}\right) \times L^{2}\left(\mathbb{R}^{3}\right)\right)$ with $(u(\tau, \tau, \omega), v(\tau, \tau, \omega))=\left(u_{0}, v_{0}\right)$. Further, the solution is continuous with respect to $\left(u_{0}, v_{0}\right)$ in $H^{1}\left(\mathbb{R}^{3}\right) \times L^{2}\left(\mathbb{R}^{3}\right)$. Sometimes, we also write the solution as $\left(u\left(t, \tau, \omega, u_{0}\right), v\left(t, \tau, \omega, v_{0}\right)\right)$ to indicate the dependence of $(u, v)$ on the initial data $\left(u_{0}, v_{0}\right)$. Notice that if $(u, v)$ is the solution of problem (3.9)-(3.11), then $(u, z)$ is the solution of problem (3.1)-(3.3) with $z(t, \tau, \omega)=v(t, \tau, \omega)+h \omega(t)$.

The following weak continuity of solutions on initial data is useful when proving the asymptotic compactness of solutions in the last section.

Lemma 3.1. Assume that $g \in L^{2}\left(\mathbb{R}^{3}\right), h \in H^{1}\left(\mathbb{R}^{3}\right)$ and (3.4)-(3.7) hold. Then the solution $(u, v)$ of problem (3.9) -(3.11) is weakly continuous with respect to the initial data $\left(u_{0}, v_{0}\right)$ in $H^{1}\left(\mathbb{R}^{3}\right) \times L^{2}\left(\mathbb{R}^{3}\right)$. That is, for $P$-a.e. $\omega \in \Omega, \tau \in \mathbb{R}$ and $t \geq$ $\tau,\left(u\left(t, \tau, \omega, u_{0, n}\right), v\left(t, \tau, \omega, v_{0, n}\right)\right)$ weakly converges to $\left(u\left(t, \tau, \omega, u_{0}\right), v\left(t, \tau, \omega, v_{0}\right)\right)$ in $H^{1}\left(\mathbb{R}^{3}\right) \times L^{2}\left(\mathbb{R}^{3}\right)$ provided $\left(u_{0, n}, v_{0, n}\right)$ weakly converges to $\left(u_{0}, v_{0}\right)$ in $H^{1}\left(\mathbb{R}^{3}\right) \times$ $L^{2}\left(\mathbb{R}^{3}\right)$.

Proof. The proof is quite standard (see, e.g., 4]) and hence is omitted here.

We now define a random dynamical system for the stochastic wave equation. Let $\Phi$ be a mapping, $\Phi$ : $\mathbb{R}^{+} \times \Omega \times H^{1}\left(\mathbb{R}^{3}\right) \times L^{2}\left(\mathbb{R}^{3}\right) \rightarrow H^{1}\left(\mathbb{R}^{3}\right) \times L^{2}\left(\mathbb{R}^{3}\right)$ given by (3.12)

$\Phi\left(t, \omega,\left(u_{0}, z_{0}\right)\right)=\left(u\left(t, 0, \omega, u_{0}\right), z\left(t, 0, \omega, z_{0}\right)\right)=\left(u\left(t, 0, \omega, u_{0}\right), v\left(t, 0, \omega, v_{0}\right)+h \omega(t)\right)$, 
for every $\left(t, \omega,\left(u_{0}, z_{0}\right)\right) \in \mathbb{R}^{+} \times \Omega \times H^{1}\left(\mathbb{R}^{3}\right) \times L^{2}\left(\mathbb{R}^{3}\right)$, where $v_{0}=z_{0}$. Then $\Phi$ is a continuous random dynamical system over $\left(\Omega, \mathcal{F}, P,\left(\theta_{t}\right)_{t \in \mathbb{R}}\right)$ on $H^{1}\left(\mathbb{R}^{3}\right) \times L^{2}\left(\mathbb{R}^{3}\right)$. It is easy to verify that $\Phi$ satisfies the following identity, for $P$-a.e. $\omega \in \Omega$ and $t \geq 0$ :

$$
\begin{aligned}
& \Phi\left(t, \theta_{-t} \omega,\left(u_{0}, z_{0}\right)\right)=\left(u\left(t, 0, \theta_{-t} \omega, u_{0}\right), z\left(t, 0, \theta_{-t} \omega, z_{0}\right)\right) \\
= & \left(u\left(0,-t, \omega, u_{0}\right), z\left(0,-t, \omega, z_{0}\right)\right)=\left(u\left(0,-t, \omega, u_{0}\right), v\left(0,-t, \omega, z_{0}\right)\right) .
\end{aligned}
$$

By the last equality of (3.13), we immediately see that the pullback asymptotic compactness for problem (3.9)-(3.11) is equivalent to the pullback asymptotic compactness for problem (3.1)-(3.3). This enables us to prove the pullback asymptotic compactness for problem (3.1)-(3.3) based on the uniform pathwise estimates on the solutions of problem (3.9)-3.11).

Throughout this paper, we always denote by $\mathcal{D}$ the collection of all tempered random subsets of $H^{1}\left(\mathbb{R}^{3}\right) \times L^{2}\left(\mathbb{R}^{3}\right)$, and we will prove that $\Phi$ has a $\mathcal{D}$-random attractor.

\section{UNIFORM ESTIMATES}

In this section, we derive uniform estimates on solutions of problem (3.9)-(3.11). These estimates are needed for proving the existence of random absorbing sets and the pullback asymptotic compactness of the random dynamical system $\Phi$.

Let $\delta>0$ be small enough such that

$$
\alpha-\delta>0, \quad \lambda+\delta^{2}-\alpha \delta>0,
$$

and denote by

$$
\sigma=\frac{1}{2} \min \left\{\alpha-\delta, \delta, \delta c_{2}\right\}
$$

where $c_{2}$ is the positive constant in (3.5).

Lemma 4.1. Assume that $g \in L^{2}\left(\mathbb{R}^{3}\right), h \in H^{1}\left(\mathbb{R}^{3}\right)$ and (3.4)-(3.7) hold. Let $B=\{B(\omega)\}_{\omega \in \Omega} \in \mathcal{D}$. Then for P-a.e. $\omega \in \Omega$, there is $T=T(B, \omega)<0$ such that for all $\tau \leq T$, the solution $\left(u\left(\cdot, \tau, \omega, u_{0}\right), v\left(\cdot, \tau, \omega, v_{0}\right)\right)$ of problem (3.9) -(3.11) with $\left(u_{0}, v_{0}\right) \in B\left(\theta_{\tau} \omega\right)$ satisfies, for every $t \in[\tau, 0]$,

$$
\left\|u\left(t, \tau, \omega, u_{0}\right)\right\|_{H^{1}\left(\mathbb{R}^{3}\right)}^{2}+\left\|v\left(t, \tau, \omega, v_{0}\right)\right\|^{2} \leq e^{-\sigma t} R(\omega)
$$

and

$$
\int_{\tau}^{t} e^{\sigma \xi}\left(\left\|u\left(\xi, \tau, \omega, u_{0}\right)\right\|_{H^{1}\left(\mathbb{R}^{3}\right)}^{2}+\left\|v\left(\xi, \tau, \omega, v_{0}\right)\right\|^{2} d \xi\right) \leq R(\omega),
$$

where $R(\omega)$ is a positive tempered random function.

Proof. Taking the inner product of (3.10) with $v$ in $L^{2}\left(\mathbb{R}^{3}\right)$, we get

$$
\begin{gathered}
\frac{1}{2} \frac{d}{d t}\|v\|^{2}+(\alpha-\delta)\|v\|^{2}+\left(\lambda+\delta^{2}-\alpha \delta\right)(u, v)-(\Delta u, v)+(f(x, u), v) \\
=(g, v)+(\delta-\alpha)(h, v) \omega(t) .
\end{gathered}
$$


By (3.9) we have

$$
\begin{gathered}
(u, v)=\frac{1}{2} \frac{d}{d t}\|u\|^{2}+\delta\|u\|^{2}-(u, h) \omega(t), \\
-(\Delta u, v)=\frac{1}{2} \frac{d}{d t}\|\nabla u\|^{2}+\delta\|\nabla u\|^{2}-(\nabla u, \nabla h) \omega(t),
\end{gathered}
$$

and

$$
(f(x, u), v)=\frac{d}{d t} \int_{\mathbb{R}^{3}} F(x, u) d x+\delta(f(x, u), u)-(f(x, u), h) \omega(t) .
$$

It follows from (4.5)-(4.8) that

$$
\begin{gathered}
\frac{d}{d t}\left(\|v\|^{2}+\left(\lambda+\delta^{2}-\alpha \delta\right)\|u\|^{2}+\|\nabla u\|^{2}+2 \int_{\mathbb{R}^{3}} F(x, u) d x\right) \\
+2(\alpha-\delta)\|v\|^{2}+2 \delta\left(\lambda+\delta^{2}-\alpha \delta\right)\|u\|^{2}+2 \delta\|\nabla u\|^{2}+2 \delta(f(x, u), u) \\
=2\left(\lambda+\delta^{2}-\alpha \delta\right)(h, u) \omega(t)+2(\nabla u, \nabla h) \omega(t)+2(f(x, u), h) \omega(t) \\
+2(g, v)+2(\delta-\alpha)(h, v) \omega(t) .
\end{gathered}
$$

We now estimate every term on the right-hand side of (4.9). For the first term, by (4.1) we have

$$
2\left(\lambda+\delta^{2}-\alpha \delta\right)(h, u) \omega(t) \leq\left(\lambda+\delta^{2}-\alpha \delta\right)\|u\|^{2}+c\|h\|^{2}|\omega(t)|^{2} .
$$

The second term on the right-hand side of (4.9) satisfies

$$
2(\nabla u, \nabla h) \omega(t) \leq \delta\|\nabla u\|^{2}+c\|\nabla h\|^{2}|\omega(t)|^{2} .
$$

For the third term on the right-hand side of (4.9), by (3.4) and (3.6), we obtain

$$
\begin{gathered}
2(f(x, u), h) \omega(t) \leq 2\left\|\phi_{1}\right\|\|h\||| \omega(t)\left|+c\left(\int_{\mathbb{R}^{3}}|u|^{\gamma+1}\right)^{\frac{\gamma}{\gamma+1}}\|h\|_{\gamma+1}\right| \omega(t) \mid \\
\leq 2\left\|\phi_{1}\right\|\|h\||\omega(t)|+c\left(\int_{\mathbb{R}^{3}}\left(F(x, u)+\phi_{3}\right)\right)^{\frac{\gamma}{\gamma+1}}\|h\|_{\gamma+1}|\omega(t)| \\
\leq 2\left\|\phi_{1}\right\|\|h\||\omega(t)|+\delta c_{2} \int_{\mathbb{R}^{3}} F(x, u) d x+\delta c_{2} \int_{\mathbb{R}^{3}} \phi_{3}(x) d x+c\|h\|_{H^{1}}^{\gamma+1}|\omega(t)|^{\gamma+1} .
\end{gathered}
$$

Similarly, by Young's inequality, the last two terms on the right-hand side of (4.9) are bounded by

$$
2|(g, v)|+2|(\delta-\alpha)(h, v) \omega(t)| \leq(\alpha-\delta)\|v\|^{2}+c\|h\|^{2}|\omega(t)|^{2}+c\|g\|^{2} .
$$

By (3.5) we also have

$$
(f(x, u), u) \geq c_{2} \int_{\mathbb{R}^{3}} F(x, u) d x+\int_{\mathbb{R}^{3}} \phi_{2}(x) d x .
$$

By (4.9)-(4.14), we find that

$$
\begin{gathered}
\frac{d}{d t}\left(\|v\|^{2}+\left(\lambda+\delta^{2}-\alpha \delta\right)\|u\|^{2}+\|\nabla u\|^{2}+2 \int_{\mathbb{R}^{3}} F(x, u) d x\right) \\
+(\alpha-\delta)\|v\|^{2}+\delta\left(\lambda+\delta^{2}-\alpha \delta\right)\|u\|^{2}+\delta\|\nabla u\|^{2}+\delta c_{2} \int_{\mathbb{R}^{3}} F(x, u) d x \\
\leq c\left(1+|\omega(t)|^{2}+|\omega(t)|^{\gamma+1}\right) .
\end{gathered}
$$


By (3.6) and (4.2) we have

$$
\delta c_{2} \int_{\mathbb{R}^{3}} F(x, u) d x \geq 2 \sigma \int_{\mathbb{R}^{3}} F(x, u) d x+\left(2 \sigma-\delta c_{2}\right) \int_{\mathbb{R}^{3}} \phi_{3}(x) d x,
$$

which along with (4.15) implies that

$$
\begin{aligned}
& \frac{d}{d t}\left(\|v\|^{2}+\left(\lambda+\delta^{2}-\alpha \delta\right)\|u\|^{2}+\|\nabla u\|^{2}+2 \int_{\mathbb{R}^{3}} F(x, u) d x\right) \\
+ & \sigma\left(\|v\|^{2}+\left(\lambda+\delta^{2}-\alpha \delta\right)\|u\|^{2}+\|\nabla u\|^{2}+2 \int_{\mathbb{R}^{3}} F(x, u) d x\right) \\
+ & \sigma\left(\|v\|^{2}+\left(\lambda+\delta^{2}-\alpha \delta\right)\|u\|^{2}+\|\nabla u\|^{2}\right) \leq c\left(1+|\omega(t)|^{2}+|\omega(t)|^{\gamma+1}\right) .
\end{aligned}
$$

Integrating (4.16) on $(\tau, t)$ with $t \leq 0$, we get

$$
\begin{gathered}
e^{\sigma t}\left(\|v(t, \tau, \omega)\|^{2}+\left(\lambda+\delta^{2}-\alpha \delta\right)\|u(t, \tau, \omega)\|^{2}+\|\nabla u(t, \tau, \omega)\|^{2}+2 \int_{\mathbb{R}^{3}} F(x, u) d x\right) \\
+\sigma \int_{\tau}^{t} e^{\sigma \xi}\left(\|v\|^{2}+\left(\lambda+\delta^{2}-\alpha \delta\right)\|u\|^{2}+\|\nabla u\|^{2}\right) d \xi \\
\leq e^{\sigma \tau}\left(\left\|v_{0}\right\|^{2}+\left(\lambda+\delta^{2}-\alpha \delta\right)\left\|u_{0}\right\|^{2}+\left\|\nabla u_{0}\right\|^{2}+2 \int_{\mathbb{R}^{3}} F\left(x, u_{0}\right) d x\right) \\
+c \int_{\tau}^{t} e^{\sigma \xi}\left(1+|\omega(\xi)|^{2}+|\omega(\xi)|^{\gamma+1}\right) d \xi .
\end{gathered}
$$

By (3.8) we have

$$
\int_{\mathbb{R}^{3}} F\left(x, u_{0}\right) d x \leq c\left(1+\left\|u_{0}\right\|^{2}+\left\|u_{0}\right\|_{H^{1}}^{\gamma+1}\right),
$$

which along with $\left(u_{0}, v_{0}\right) \in B\left(\theta_{\tau} \omega\right)$ implies that

$$
\begin{aligned}
& e^{\sigma \tau}\left(\left\|v_{0}\right\|^{2}+\left(\lambda+\delta^{2}-\alpha \delta\right)\left\|u_{0}\right\|^{2}+\left\|\nabla u_{0}\right\|^{2}+2 \int_{\mathbb{R}^{3}} F\left(x, u_{0}\right) d x\right) \\
& \quad \leq c e^{\sigma \tau}\left(1+\left\|v_{0}\right\|^{2}+\left\|u_{0}\right\|_{H^{1}}^{2}+\left\|u_{0}\right\|_{H^{1}}^{\gamma+1}\right) \rightarrow 0 \quad \text { as } \tau \rightarrow-\infty .
\end{aligned}
$$

Therefore, there exists $T=T(B, \omega)<0$ such that for all $\tau \leq T$,

$$
e^{\sigma \tau}\left(\left\|v_{0}\right\|^{2}+\left(\lambda+\delta^{2}-\alpha \delta\right)\left\|u_{0}\right\|^{2}+\left\|\nabla u_{0}\right\|^{2}+2 \int_{\mathbb{R}^{3}} F\left(x, u_{0}\right) d x\right) \leq r(\omega),
$$

where

$$
r(\omega)=\int_{-\infty}^{0} e^{\sigma \xi}\left(1+|\omega(\xi)|^{2}+|\omega(\xi)|^{\gamma+1}\right) d \xi .
$$

Notice that $r(\omega)$ is well defined since $\omega(\xi)$ has at most linear growth rate as $|\xi| \rightarrow \infty$. By (4.17) and (4.19) we obtain that, for all $\tau \leq T$ and $t \in[\tau, 0]$,

$$
\begin{aligned}
& e^{\sigma t}\left(\|v(t, \tau, \omega)\|^{2}+\left(\lambda+\delta^{2}-\alpha \delta\right)\|u(t, \tau, \omega)\|^{2}+\|\nabla u(t, \tau, \omega)\|^{2}+2 \int_{\mathbb{R}^{3}} F(x, u) d x\right) \\
& (4.20) \quad+\int_{\tau}^{t} e^{\sigma \xi}\left(\|v\|^{2}+\left(\lambda+\delta^{2}-\alpha \delta\right)\|u\|^{2}+\|\nabla u\|^{2}\right) d \xi \leq c(1+r(\omega)) .
\end{aligned}
$$


By (3.6), we find that, for all $t \leq 0$,

$$
-2 e^{\sigma t} \int_{\mathbb{R}^{3}} F(x, u) d x \leq 2 e^{\sigma t} \int_{\mathbb{R}^{3}} \phi_{3}(x) d x \leq 2 \int_{\mathbb{R}^{3}}\left|\phi_{3}(x)\right| d x .
$$

By (4.20) and (4.21) we have that, for all $\tau \leq T$ and $t \in[\tau, 0]$,

$$
\begin{aligned}
& e^{\sigma t}\left(\|v(t, \tau, \omega)\|^{2}+\left(\lambda+\delta^{2}-\alpha \delta\right)\|u(t, \tau, \omega)\|^{2}+\|\nabla u(t, \tau, \omega)\|^{2}\right) \\
& +\int_{\tau}^{t} e^{\sigma \xi}\left(\|v\|^{2}+\left(\lambda+\delta^{2}-\alpha \delta\right)\|u\|^{2}+\|\nabla u\|^{2}\right) d \xi \leq c(1+r(\omega)),
\end{aligned}
$$

which implies (4.3) and (4.4) with $R(\omega)=c(1+r(\omega))$. Next we show that $R(\omega)$ is tempered; that is, for every $\beta>0$, we want to prove

$$
e^{\beta \tau} R\left(\theta_{\tau} \omega\right) \rightarrow 0 \quad \text { as } \quad \tau \rightarrow-\infty .
$$

Without loss of generality, we now assume $\beta \leq \sigma$. Then we have

$$
\begin{aligned}
e^{\beta \tau} R\left(\theta_{\tau} \omega\right) & =c e^{\beta \tau}+c e^{\beta \tau} \int_{-\infty}^{0} e^{\sigma \xi}\left(\left|\left(\theta_{\tau} \omega\right)(\xi)\right|^{2}+\left|\left(\theta_{\tau} \omega\right)(\xi)\right|^{\gamma+1}\right) d \xi \\
\leq & c e^{\beta \tau}+c e^{\beta \tau} \int_{-\infty}^{0} e^{\beta \xi}\left(\left|\left(\theta_{\tau} \omega\right)(\xi)\right|^{2}+\left|\left(\theta_{\tau} \omega\right)(\xi)\right|^{\gamma+1}\right) d \xi \\
\leq & c e^{\beta \tau}+c e^{\beta \tau} \int_{-\infty}^{0} e^{\beta \xi}\left(|\omega(\tau)|^{2}+|\omega(\tau)|^{\gamma+1}\right) d \xi \\
& +c e^{\beta \tau} \int_{-\infty}^{0} e^{\beta \xi}\left(|\omega(\tau+\xi)|^{2}+|\omega(\tau+\xi)|^{\gamma+1}\right) d \xi \\
& \leq c e^{\beta \tau}+\frac{c}{\beta} e^{\beta \tau}\left(|\omega(\tau)|^{2}+|\omega(\tau)|^{\gamma+1}\right)+c \int_{-\infty}^{\tau} e^{\beta s}\left(|\omega(s)|^{2}+|\omega(s)|^{\gamma+1}\right) d s .
\end{aligned}
$$

Then (4.22) follows from (4.23) since $\omega$ has at most linear growth rate at infinity. This completes the proof.

We now derive an energy equation for problem (3.9)-(3.11). To this end, denote by, for $(u, v) \in H^{1}\left(\mathbb{R}^{3}\right) \times L^{2}\left(\mathbb{R}^{3}\right)$,

$$
E(u, v)=\|v\|^{2}+\left(\lambda+\delta^{2}-\alpha \delta\right)\|u\|^{2}+\|\nabla u\|^{2}+2 \int_{\mathbb{R}^{3}} F(x, u) d x,
$$

and

$$
\begin{aligned}
& \Psi\left(u\left(t, \tau, \omega, u_{0}\right), v\left(t, \tau, \omega, v_{0}\right)\right) \\
& =-2(\alpha-\delta-2 \sigma)\|v\|^{2}-2(\delta-2 \sigma)\left(\lambda+\delta^{2}-\alpha \delta\right)\|u\|^{2}-2(\delta-2 \sigma)\|\nabla u\|^{2} \\
& \quad+8 \sigma \int_{\mathbb{R}^{3}} F(x, u) d x-2 \delta \int_{\mathbb{R}^{3}} f(x, u) u d x+2\left(\lambda+\delta^{2}-\alpha \delta\right)(u, h) \omega(t) \\
& \quad+2(\nabla u, \nabla h) \omega(t)+2 \omega(t) \int_{\mathbb{R}^{3}} f(x, u) h(x) d x+2(g, v)+2(\delta-\alpha)(v, h) \omega(t) .
\end{aligned}
$$


Then it follows from (4.9) that

$$
\frac{d}{d t} E+4 \sigma E=\Psi
$$

Integrating (4.26) on $(\tau, t)$ we get

$$
\begin{aligned}
& E\left(u\left(t, \tau, \omega, u_{0}\right), v\left(t, \tau, \omega, v_{0}\right)\right) \\
& \quad=e^{-4 \sigma(t-\tau)} E\left(u_{0}, v_{0}\right)+\int_{\tau}^{t} e^{4 \sigma(\xi-t)} \Psi\left(u\left(\xi, \tau, \omega, u_{0}\right), v\left(\xi, \tau, \omega, v_{0}\right)\right) d \xi .
\end{aligned}
$$

The energy equation (4.27) will be used to prove the pullback asymptotic compactness of solutions in the last section.

In what follows, we derive uniform estimates on the tails of solutions when $x$ and $t$ approach infinity. These estimates will be used to overcome the difficulty caused by the noncompactness of embeddings $H^{1}\left(\mathbb{R}^{3}\right) \hookrightarrow L^{p}\left(\mathbb{R}^{3}\right)$ for $p \leq 6$ and are crucial for proving the pullback asymptotic compactness of the random dynamical system. Given $k \geq 1$, denote by $Q_{k}=\left\{x \in \mathbb{R}^{3}:|x|<k\right\}$ and $\mathbb{R}^{3} \backslash Q_{k}$ the complement of $Q_{k}$.

Lemma 4.2. Assume that $g \in L^{2}\left(\mathbb{R}^{3}\right), h \in H^{1}\left(\mathbb{R}^{3}\right)$ and (3.4)-(3.7) hold. Let $B=\{B(\omega)\}_{\omega \in \Omega} \in \mathcal{D}$. Then for every $\epsilon>0$ and P-a.e. $\omega \in \Omega$, there exist $T=T(B, \omega, \epsilon)<0$ and $k_{0}=k_{0}(\omega, \epsilon)>0$ such that for all $\tau \leq T$ and $k \geq k_{0}$, the solution $\left(u\left(\cdot, \tau, \omega, u_{0}\right), v\left(\cdot, \tau, \omega, v_{0}\right)\right)$ of problem (3.9)-(3.11) with $\left(u_{0}, v_{0}\right) \in B\left(\theta_{\tau} \omega\right)$ satisfies, for any $t \in[\tau, 0]$,

$$
\int_{\mathbb{R}^{3} \backslash Q_{k}}\left(\left|u\left(t, \tau, \omega, u_{0}\right)\right|^{2}+\left|\nabla u\left(t, \tau, \omega, u_{0}\right)\right|^{2}+\left|v\left(t, \tau, \omega, v_{0}\right)\right|^{2}\right) d x \leq \epsilon e^{-\sigma t}
$$

Proof. We will use a cutoff technique as in [35] for deterministic parabolic equations. Take a smooth function $\rho$ such that $0 \leq \rho \leq 1$ for all $s \in \mathbb{R}$ and

$$
\rho(s)=\left\{\begin{array}{lll}
0, & \text { if } \quad|s|<1 \\
1, & \text { if } \quad|s|>2
\end{array}\right.
$$

Then there is a positive constant $c$ such that $\left|\rho^{\prime}(s)\right| \leq c$ for all $s \in \mathbb{R}$.

Taking the inner product of (3.10) with $\rho\left(\frac{|x|^{2}}{k^{2}}\right) v$ in $L^{2}\left(\mathbb{R}^{3}\right)$, we get

$$
\begin{aligned}
& \frac{1}{2} \frac{d}{d t} \int_{\mathbb{R}^{3}} \rho\left(\frac{|x|^{2}}{k^{2}}\right)|v|^{2} d x+(\alpha-\delta) \int_{\mathbb{R}^{3}} \rho\left(\frac{|x|^{2}}{k^{2}}\right)|v|^{2} d x \\
+ & \left(\lambda+\delta^{2}-\alpha \delta\right) \int_{\mathbb{R}^{3}} \rho\left(\frac{|x|^{2}}{k^{2}}\right) u v d x-\int_{\mathbb{R}^{3}} \rho\left(\frac{|x|^{2}}{k^{2}}\right) v \Delta u d x \\
+ & \int_{\mathbb{R}^{3}} \rho\left(\frac{|x|^{2}}{k^{2}}\right) f(x, u) v d x=\int_{\mathbb{R}^{3}} \rho\left(\frac{|x|^{2}}{k^{2}}\right)(g v+(\delta-\alpha) v h \omega(t)) d x .
\end{aligned}
$$


By (3.9) we find that

$$
\begin{gathered}
\int_{\mathbb{R}^{3}} \rho\left(\frac{|x|^{2}}{k^{2}}\right) u v d x=\frac{1}{2} \frac{d}{d t} \int_{\mathbb{R}^{3}} \rho\left(\frac{|x|^{2}}{k^{2}}\right)|u|^{2} d x \\
+\delta \int_{\mathbb{R}^{3}} \rho\left(\frac{|x|^{2}}{k^{2}}\right)|u|^{2} d x-\int_{\mathbb{R}^{3}} \rho\left(\frac{|x|^{2}}{k^{2}}\right) u h \omega(t) d x, \\
-\int_{\mathbb{R}^{3}} \rho\left(\frac{|x|^{2}}{k^{2}}\right) v \Delta u d x=\int_{\mathbb{R}^{3}} \nabla u \frac{2 x}{k^{2}} \rho^{\prime}\left(\frac{|x|^{2}}{k^{2}}\right) v d x+\frac{1}{2} \frac{d}{d t} \int_{\mathbb{R}^{3}} \rho\left(\frac{|x|^{2}}{k^{2}}\right)|\nabla u|^{2} d x \\
+\delta \int_{\mathbb{R}^{3}} \rho\left(\frac{|x|^{2}}{k^{2}}\right)|\nabla u|^{2} d x-\int_{\mathbb{R}^{3}} \rho\left(\frac{|x|^{2}}{k^{2}}\right) \nabla u \nabla h \omega(t) d x,
\end{gathered}
$$

and

$$
\begin{aligned}
& \int_{\mathbb{R}^{3}} \rho\left(\frac{|x|^{2}}{k^{2}}\right) f(x, u) v d x=\frac{d}{d t} \int_{\mathbb{R}^{3}} \rho\left(\frac{|x|^{2}}{k^{2}}\right) F(x, u) d x \\
& +\delta \int_{\mathbb{R}^{3}} \rho\left(\frac{|x|^{2}}{k^{2}}\right) f(x, u) u d x-\int_{\mathbb{R}^{3}} \rho\left(\frac{|x|^{2}}{k^{2}}\right) f(x, u) h \omega(t) d x .
\end{aligned}
$$

It follows from (4.30)- (4.33) that

$$
\begin{gathered}
\quad \frac{d}{d t} \int_{\mathbb{R}^{3}} \rho\left(\frac{|x|^{2}}{k^{2}}\right)\left(|v|^{2}+\left(\lambda+\delta^{2}-\alpha \delta\right)|u|^{2}+|\nabla u|^{2}+2 F(x, u)\right) d x \\
+\int_{\mathbb{R}^{3}} \rho\left(\frac{|x|^{2}}{k^{2}}\right)\left(2(\alpha-\delta)|v|^{2}+2 \delta\left(\lambda+\delta^{2}-\alpha \delta\right)|u|^{2}+2 \delta|\nabla u|^{2}+2 \delta f(x, u) u\right) d x \\
=2\left(\lambda+\delta^{2}-\alpha \delta\right) \int_{\mathbb{R}^{3}} \rho\left(\frac{|x|^{2}}{k^{2}}\right) h u \omega(t) d x-4 \int_{\mathbb{R}^{3}} \rho^{\prime}\left(\frac{|x|^{2}}{k^{2}}\right) v \nabla u \frac{x}{k^{2}} d x \\
+2 \int_{\mathbb{R}^{3}} \rho\left(\frac{|x|^{2}}{k^{2}}\right) f(x, u) h \omega(t) d x+2 \int_{\mathbb{R}^{3}} \rho\left(\frac{|x|^{2}}{k^{2}}\right) \nabla u \nabla h \omega(t) d x \\
+2 \int_{\mathbb{R}^{3}} \rho\left(\frac{|x|^{2}}{k^{2}}\right)(g v+(\delta-\alpha) h v \omega(t)) d x .
\end{gathered}
$$

By (3.5) we have

$$
\int_{\mathbb{R}^{3}} \rho\left(\frac{|x|^{2}}{k^{2}}\right) f(x, u) u d x \geq c_{2} \int_{\mathbb{R}^{3}} \rho\left(\frac{|x|^{2}}{k^{2}}\right) F(x, u) d x+\int_{\mathbb{R}^{3}} \rho\left(\frac{|x|^{2}}{k^{2}}\right) \phi_{2}(x) d x .
$$

By (3.4) and (3.6) as in (4.12), we also have

$$
\begin{gathered}
2 \int_{\mathbb{R}^{3}} \rho\left(\frac{|x|^{2}}{k^{2}}\right) f(x, u) h \omega(t) d x \leq \int_{\mathbb{R}^{3}} \rho\left(\frac{|x|^{2}}{k^{2}}\right)\left|\phi_{1}\right|^{2} d x \\
+c \int_{\mathbb{R}^{3}} \rho\left(\frac{|x|^{2}}{k^{2}}\right)|h|^{2}|\omega(t)|^{2} d x \\
+\delta c_{2} \int_{\mathbb{R}^{3}} \rho\left(\frac{|x|^{2}}{k^{2}}\right)\left(F(x, u)+\phi_{3}(x)\right) d x+c \int_{\mathbb{R}^{3}} \rho\left(\frac{|x|^{2}}{k^{2}}\right)|h|^{\gamma+1}|\omega(t)|^{\gamma+1} d x .
\end{gathered}
$$


By the definition of $\rho$ in (4.29) we have

$$
\int_{\mathbb{R}^{3}}\left|\rho^{\prime}\left(\frac{|x|^{2}}{k^{2}}\right) v \nabla u \frac{x}{k^{2}}\right| d x \leq \int_{k \leq|x| \leq \sqrt{2} k}\left|\rho^{\prime}\right||v||\nabla u| \frac{|x|}{k^{2}} d x \leq \frac{c}{k}\left(\|\nabla u\|^{2}+\|v\|^{2}\right) .
$$

Using Young's inequality to estimate the remaining terms on on the right-hand side of (4.34), by (4.35)-(4.37), we find that

$$
\begin{gathered}
\frac{d}{d t} \int_{\mathbb{R}^{3}} \rho\left(\frac{|x|^{2}}{k^{2}}\right)\left(|v|^{2}+\left(\lambda+\delta^{2}-\alpha \delta\right)|u|^{2}+|\nabla u|^{2}+2 F(x, u)\right) d x \\
+\int_{\mathbb{R}^{3}} \rho\left(\frac{|x|^{2}}{k^{2}}\right)\left((\alpha-\delta)|v|^{2}+\delta\left(\lambda+\delta^{2}-\alpha \delta\right)|u|^{2}+\delta|\nabla u|^{2}+\delta c_{2} F(x, u)\right) d x \\
\leq \frac{c}{k}\left(\|\nabla u\|^{2}+\|v\|^{2}\right)+c|\omega(t)|^{2} \int_{\mathbb{R}^{3}} \rho\left(\frac{|x|^{2}}{k^{2}}\right)\left(|h|^{2}+|\nabla h|^{2}\right) d x \\
\quad+c \int_{\mathbb{R}^{3}} \rho\left(\frac{|x|^{2}}{k^{2}}\right)\left(\left|\phi_{1}\right|^{2}+\left|\phi_{2}\right|+\left|\phi_{3}\right|+|g|^{2}+|\omega(t)|^{\gamma+1}|h|^{\gamma+1}\right) d x .
\end{gathered}
$$

For the last two terms on the right-hand side of (4.38), we find that there exists $k_{1}=k_{1}(\epsilon) \geq 1$ such that for all $k \geq k_{1}$,

$$
\begin{gathered}
c|\omega(t)|^{2} \int_{\mathbb{R}^{3}} \rho\left(\frac{|x|^{2}}{k^{2}}\right)\left(|h|^{2}+|\nabla h|^{2}\right) d x \\
+c \int_{\mathbb{R}^{3}} \rho\left(\frac{|x|^{2}}{k^{2}}\right)\left(\left|\phi_{1}\right|^{2}+\left|\phi_{2}\right|+\left|\phi_{3}\right|+|g|^{2}+|\omega(t)|^{\gamma+1}|h|^{\gamma+1}\right) d x \\
=c|\omega(t)|^{2} \int_{|x| \geq k} \rho\left(\frac{|x|^{2}}{k^{2}}\right)\left(|h|^{2}+|\nabla h|^{2}\right) d x \\
+c \int_{|x| \geq k} \rho\left(\frac{|x|^{2}}{k^{2}}\right)\left(\left|\phi_{1}\right|^{2}+\left|\phi_{2}\right|+\left|\phi_{3}\right|+|g|^{2}+|\omega(t)|^{\gamma+1}|h|^{\gamma+1}\right) d x \\
\leq c|\omega(t)|^{2} \int_{|x| \geq k}\left(|h|^{2}+|\nabla h|^{2}\right) d x \\
+c \int_{|x| \geq k}\left(\left|\phi_{1}\right|^{2}+\left|\phi_{2}\right|+\left|\phi_{3}\right|+|g|^{2}+|\omega(t)|^{\gamma+1}|h|^{\gamma+1}\right) d x \\
\leq c \epsilon\left(1+|\omega(t)|^{2}+|\omega(t)|^{\gamma+1}\right),
\end{gathered}
$$

where we have used the fact that $\phi_{1}, g \in L^{2}\left(\mathbb{R}^{n}\right), \phi_{2}, \phi_{3} \in L^{1}\left(\mathbb{R}^{n}\right), h \in H^{1}\left(\mathbb{R}^{n}\right)$, and the embedding $H^{1}\left(\mathbb{R}^{3}\right) \hookrightarrow L^{\gamma+1}\left(\mathbb{R}^{3}\right)$ with $\gamma \leq 3$. It follows from (4.38)-(4.39) that, for all $k \geq k_{1}$,

$$
\begin{gathered}
\frac{d}{d t} \int_{\mathbb{R}^{3}} \rho\left(\frac{|x|^{2}}{k^{2}}\right)\left(|v|^{2}+\left(\lambda+\delta^{2}-\alpha \delta\right)|u|^{2}+|\nabla u|^{2}+2 F(x, u)\right) d x \\
+\int_{\mathbb{R}^{3}} \rho\left(\frac{|x|^{2}}{k^{2}}\right)\left((\alpha-\delta)|v|^{2}+\delta\left(\lambda+\delta^{2}-\alpha \delta\right)|u|^{2}+\delta|\nabla u|^{2}+\delta c_{2} F(x, u)\right) d x \\
\leq \frac{c}{k}\left(\|\nabla u\|^{2}+\|v\|^{2}\right)+c \epsilon\left(1+|\omega(t)|^{2}+|\omega(t)|^{\gamma+1}\right) .
\end{gathered}
$$


By (3.6), (4.2) and (4.40) we find that for all $k \geq k_{1}$,

$$
\begin{gathered}
\left.\frac{d}{d t} \int_{\mathbb{R}^{3}} \rho\left(\frac{|x|^{2}}{k^{2}}\right)\left(|v|^{2}+\left(\lambda+\delta^{2}-\alpha \delta\right)|u|^{2}+|\nabla u|^{2}+2 F(x, u)\right) d x\right] \\
+\sigma \int_{\mathbb{R}^{3}} \rho\left(\frac{|x|^{2}}{k^{2}}\right)\left(|v|^{2}+\left(\lambda+\delta^{2}-\alpha \delta\right)|u|^{2}+|\nabla u|^{2}+2 F(x, u)\right) d x \\
\leq \frac{c}{k}\left(\|\nabla u\|^{2}+\|v\|^{2}\right)+c \epsilon\left(1+|\omega(t)|^{2}+|\omega(t)|^{\gamma+1}\right) .
\end{gathered}
$$

Integrating (4.41) on $(\tau, t)$ with $t \leq 0$, by Lemma 4.1 we find that, for all $k \geq k_{1}$,

$$
\begin{gathered}
e^{\sigma t} \int_{\mathbb{R}^{3}} \rho\left(\frac{|x|^{2}}{k^{2}}\right)\left(|v(t, \tau, \omega)|^{2}+\left(\lambda+\delta^{2}-\alpha \delta\right)|u(t, \tau, \omega)|^{2}+|\nabla u(t, \tau, \omega)|^{2}+2 F(x, u)\right) \\
\leq e^{\sigma \tau} \int_{\mathbb{R}^{3}} \rho\left(\frac{|x|^{2}}{k^{2}}\right)\left(\left|v_{0}\right|^{2}+\left(\lambda+\delta^{2}-\alpha \delta\right)\left|u_{0}\right|^{2}+\left|\nabla u_{0}\right|^{2}+2 F\left(x, u_{0}\right)\right) d x \\
+\frac{c}{k} \int_{\tau}^{t} e^{\sigma \xi}\left(\|\nabla u(\xi)\|^{2}+\|v(\xi)\|^{2}\right) d \xi+c \epsilon \int_{\tau}^{t} e^{\sigma \xi}\left(|\omega(\xi)|^{2}+|\omega(\xi)|^{\gamma+1}\right) d \xi+c \epsilon \\
\leq e^{\sigma \tau} \int_{\mathbb{R}^{3}} \rho\left(\frac{|x|^{2}}{k^{2}}\right)\left(\left|v_{0}\right|^{2}+\left(\lambda+\delta^{2}-\alpha \delta\right)\left|u_{0}\right|^{2}+\left|\nabla u_{0}\right|^{2}+2 F\left(x, u_{0}\right)\right) d x \\
\quad+\frac{c}{k} R(\omega)+c \epsilon \int_{-\infty}^{0} e^{\sigma \xi}\left(|\omega(\xi)|^{2}+|\omega(\xi)|^{\gamma+1}\right) d \xi+c \epsilon,
\end{gathered}
$$

where $R(\omega)$ is the positive tempered random function in Lemma 4.1, As in (4.18), the first term on the right-hand side of (4.42) goes to zero as $\tau \rightarrow-\infty$. Hence, there exists $T=T(B, \omega, \epsilon)<0$ such that for all $\tau \leq T$,

$$
e^{\sigma \tau} \int_{\mathbb{R}^{3}} \rho\left(\frac{|x|^{2}}{k^{2}}\right)\left(\left|v_{0}\right|^{2}+\left(\lambda+\delta^{2}-\alpha \delta\right)\left|u_{0}\right|^{2}+\left|\nabla u_{0}\right|^{2}+2 F\left(x, u_{0}\right)\right) d x \leq \epsilon .
$$

By (4.42)-(4.43), there exists $k_{2}(\epsilon) \geq k_{1}(\epsilon)$ such that for all $\tau \leq T$ and $k \geq k_{2}$,

$$
\begin{aligned}
e^{\sigma t} \int_{\mathbb{R}^{3}} \rho\left(\frac{|x|^{2}}{k^{2}}\right)\left(|v(t, \tau, \omega)|^{2}+(\right. & \left.+\delta^{2}-\alpha \delta\right)|u(t, \tau, \omega)|^{2} \\
& \left.+|\nabla u(t, \tau, \omega)|^{2}+2 F(x, u)\right) d x \leq \epsilon c r(\omega),
\end{aligned}
$$

where $r(\omega)=1+R(\omega)+\int_{-\infty}^{0} e^{\sigma \xi}\left(|\omega(\xi)|^{2}+|\omega(\xi)|^{\gamma+1}\right) d \xi$. By (3.6) we have, for $t \leq 0$,

$$
\begin{aligned}
-2 e^{\sigma t} \int_{\mathbb{R}^{3}} \rho\left(\frac{|x|^{2}}{k^{2}}\right) F(x, u) d x & \leq 2 e^{\sigma t} \int_{\mathbb{R}^{3}} \rho\left(\frac{|x|^{2}}{k^{2}}\right) \phi_{3}(x) d x \leq 2 \int_{|x| \geq k} \rho\left(\frac{|x|^{2}}{k^{2}}\right) \phi_{3}(x) d x \\
& \leq 2 \int_{|x| \geq k}\left|\phi_{3}(x)\right| d x .
\end{aligned}
$$

Since $\phi_{3} \in L^{1}\left(\mathbb{R}^{3}\right)$, there is $k_{3}=k_{3}(\epsilon) \geq k_{2}$ such that for all $k \geq k_{3}$, the right-hand side of the above is bounded by $\epsilon$. Hence we have, for all $k \geq k_{3}$ and $t \leq 0$,

$$
-2 e^{\sigma t} \int_{\mathbb{R}^{3}} \rho\left(\frac{|x|^{2}}{k^{2}}\right) F(x, u) d x \leq \epsilon .
$$


By (4.44)-(4.45) we get that, for all $\tau \leq T, t \in[\tau, 0]$ and $k \geq k_{3}$,

$$
e^{\sigma t} \int_{\mathbb{R}^{3}}\left(|v(t, \tau, \omega)|^{2}+\left(\lambda+\delta^{2}-\alpha \delta\right)|u(t, \tau, \omega)|^{2}+|\nabla u(t, \tau, \omega)|^{2}\right) d x \leq \epsilon+c \epsilon r(\omega) .
$$

By the definition of $\rho$ in (4.29), we finally obtain that, for all $\tau \leq T, t \in[\tau, 0]$ and $k \geq k_{3}$,

$$
\begin{aligned}
& e^{\sigma t} \int_{|x| \geq \sqrt{2} k}\left(|v(t, \tau, \omega)|^{2}+\left(\lambda+\delta^{2}-\alpha \delta\right)|u(t, \tau, \omega)|^{2}+|\nabla u(t, \tau, \omega)|^{2}\right) d x \\
& \quad \leq e^{\sigma t} \int_{\mathbb{R}^{3}} \rho\left(\frac{|x|^{2}}{k^{2}}\right)\left(|v(t, \tau, \omega)|^{2}+\left(\lambda+\delta^{2}-\alpha \delta\right)|u(t, \tau, \omega)|^{2}+|\nabla u(t, \tau, \omega)|^{2}\right) d x \\
& \quad \leq \epsilon+c \epsilon r(\omega),
\end{aligned}
$$

which completes the proof.

\section{RANDOM ATTRACTORS}

In this section, we prove existence of a $\mathcal{D}$-random attractor for the stochastic wave equation on $\mathbb{R}^{3}$. We first show that the random dynamical system $\Phi$ has a closed random absorbing set in $\mathcal{D}$, and then prove that $\Phi$ is $\mathcal{D}$-pullback asymptotically compact.

By Lemma 4.1 we find that for every $B=\{B(\omega)\}_{\omega \in \Omega} \in \mathcal{D}$, and $P$-a.e. $\omega \in \Omega$, there is $T=T(B, \omega)<0$ such that for all $\tau \leq T$, the solution $(u, v)$ of problem (3.9)-(3.11) with $\left(u_{0}, v_{0}\right) \in B\left(\theta_{\tau} \omega\right)$ satisfies

$$
\left\|u\left(0, \tau, \omega, u_{0}\right)\right\|_{H^{1}\left(\mathbb{R}^{3}\right)}^{2}+\left\|v\left(0, \tau, \omega, v_{0}\right)\right\|^{2} \leq R(\omega),
$$

where $R(\omega)$ is the positive tempered random function in Lemma 4.1 Since $z\left(t, \tau, \omega, z_{0}\right)=v\left(t, \tau, \omega, v_{0}\right)+h \omega(t)$ with $z_{0}=v_{0}+h \omega(\tau)$, it follows from (5.1) that $\left(u\left(t, \tau, \omega, u_{0}\right), z\left(t, \tau, \omega, z_{0}\right)\right)$ with $\left(u_{0}, z_{0}\right) \in B\left(\theta_{\tau} \omega\right)$ satisfies, for all $\tau \leq T$,

$\left\|u\left(0, \tau, \omega, u_{0}\right)\right\|_{H^{1}}^{2}+\left\|z\left(0, \tau, \omega, z_{0}\right)\right\|^{2}=\left\|u\left(0, \tau, \omega, u_{0}\right)\right\|_{H^{1}}^{2}+\left\|v\left(0, \tau, \omega, v_{0}\right)\right\|^{2} \leq R(\omega)$, which along with (3.13) implies that, for all $t \geq-T$,

$$
\left\|\Phi\left(t, \theta_{-t} \omega,\left(u_{0}, z_{0}\right)\right)\right\|_{H^{1} \times L^{2}}^{2}=\left\|u\left(0,-t, \omega, u_{0}\right)\right\|_{H^{1}}^{2}+\left\|v\left(0,-t, \omega, v_{0}\right)\right\|^{2} \leq R(\omega) .
$$

Denote by

$$
\tilde{B}(\omega)=\left\{(u, z) \in H^{1}\left(\mathbb{R}^{3}\right) \times L^{2}\left(\mathbb{R}^{3}\right):\|u\|_{H^{1}}^{2}+\|z\|^{2} \leq R(\omega)\right\} .
$$

Then (5.2) shows that $\tilde{B}=\{\tilde{B}(\omega)\}_{\omega \in \Omega}$ is a closed random absorbing set for $\Phi$ in $\mathcal{D}$. Next, we show the pullback asymptotic compactness of $(u, v)$, which is needed to prove the asymptotic compactness of $\Phi$.

Lemma 5.1. Assume that $g \in L^{2}\left(\mathbb{R}^{3}\right), h \in H^{1}\left(\mathbb{R}^{3}\right)$ and (3.4) - (3.7) hold. Then, for $P$-a.e. $\omega \in \Omega$, the sequence $\left\{\left(u\left(0,-t_{n}, \omega, u_{0, n}\right), v\left(0,-t_{n}, \omega, v_{0, n}\right)\right)\right\}$ has a convergent subsequence in $H^{1}\left(\mathbb{R}^{3}\right) \times L^{2}\left(\mathbb{R}^{3}\right)$ provided $t_{n} \rightarrow \infty$ and $\left(u_{0, n}, v_{0, n}\right) \in B\left(\theta_{-t_{n}} \omega\right)$ with $B=\{B(\omega)\}_{\omega \in \Omega} \in \mathcal{D}$.

Proof. Since $t_{n} \rightarrow \infty$, it follows from (5.1) that there exists $N_{1}=N_{1}(B, \omega)>0$ such that for all $n \geq N_{1}$,

$$
\left\|u\left(0,-t_{n}, \omega, u_{0, n}\right)\right\|_{H^{1}}^{2}+\left\|v\left(0,-t_{n}, \omega, v_{0, n}\right)\right\|^{2} \leq R(\omega) .
$$


Notice that (5.4) implies that there exists $(\tilde{u}, \tilde{v}) \in H^{1}\left(\mathbb{R}^{3}\right) \times L^{2}\left(\mathbb{R}^{3}\right)$ such that, up to a subsequence,

$$
\left(u\left(0,-t_{n}, \omega, u_{0, n}\right), v\left(0,-t_{n}, \omega, v_{0, n}\right)\right) \rightarrow(\tilde{u}, \tilde{v}) \text { weakly in } H^{1}\left(\mathbb{R}^{3}\right) \times L^{2}\left(\mathbb{R}^{3}\right) .
$$

By (5.5) we find that

$$
\liminf _{n \rightarrow \infty}\left\|\left(u\left(0,-t_{n}, \omega, u_{0, n}\right), v\left(0,-t_{n}, \omega, v_{0, n}\right)\right)\right\|_{H^{1} \times L^{2}} \geq\|(\tilde{u}, \tilde{v})\|_{H^{1} \times L^{2}} .
$$

Next we prove that (5.5) is actually a strong convergence. To this end, taking (5.6) into account, we only need to show that

$$
\limsup _{n \rightarrow \infty}\left\|\left(u\left(0,-t_{n}, \omega, u_{0, n}\right), v\left(0,-t_{n}, \omega, v_{0, n}\right)\right)\right\|_{H^{1} \times L^{2}} \leq\|(\tilde{u}, \tilde{v})\|_{H^{1} \times L^{2}} .
$$

We now prove (5.7) by the energy equation (4.27). It follows from Lemma 4.1 that there exists $N_{2}=N_{2}(B, \omega)>0$ such that for all $n \geq N_{2}$,

$$
\left\|u\left(t,-t_{n}, \omega, u_{0, n}\right)\right\|_{H^{1}\left(\mathbb{R}^{3}\right)}^{2}+\left\|v\left(t,-t_{n}, \omega, v_{0, n}\right)\right\|^{2} \leq e^{-\sigma t} R(\omega),
$$

where $-t_{n} \leq t \leq 0$. Given $m>0$, let $N_{3}=N_{3}(m)>0$ be large enough such that $t_{n} \geq m$ for all $n \geq N_{3}$. Denote by $N_{4}=\max \left\{N_{2}, N_{3}\right\}$. Then by (5.8) we get that, for all $n \geq N_{4}$,

$$
\left\|u\left(-m,-t_{n}, \omega, u_{0, n}\right)\right\|_{H^{1}\left(\mathbb{R}^{3}\right)}^{2}+\left\|v\left(-m,-t_{n}, \omega, v_{0, n}\right)\right\|^{2} \leq e^{\sigma m} R(\omega) .
$$

By a diagonal procedure, we conclude from (5.9) that there exist a sequence $\left\{\tilde{u}_{m}, \tilde{v}_{m}\right\}_{m=1}^{\infty}$ in $H^{1}\left(\mathbb{R}^{3}\right) \times L^{2}\left(\mathbb{R}^{3}\right)$ and a subsequence of $\left\{\left(t_{n}, u_{0, n}, v_{0, n}\right)\right\}_{n=1}^{\infty}$ (not relabeled) such that for every positive integer $m$, when $n \rightarrow \infty$,

$\left(u\left(-m,-t_{n}, \omega, u_{0, n}\right), v\left(-m,-t_{n}, \omega, v_{0, n}\right)\right) \rightarrow\left(\tilde{u}_{m}, \tilde{v}_{m}\right)$ weakly in $H^{1}\left(\mathbb{R}^{3}\right) \times L^{2}\left(\mathbb{R}^{3}\right)$.

Notice that

$$
\begin{gathered}
\left(u\left(0,-t_{n}, \omega, u_{0, n}\right), v\left(0,-t_{n}, \omega, v_{0, n}\right)\right) \\
=\left(u\left(0,-m, \omega, u\left(-m,-t_{n}, \omega, u_{0, n}\right)\right), v\left(0,-m, \omega, v\left(-m,-t_{n}, \omega, v_{0, n}\right)\right)\right),
\end{gathered}
$$

which along with (5.10) and Lemma 3.1 implies that, for every positive integer $m$, when $n \rightarrow \infty$,

$$
u\left(0,-t_{n}, \omega, u_{0, n}\right) \rightarrow u\left(0,-m, \omega, \tilde{u}_{m}\right) \quad \text { weakly in } \quad H^{1}\left(\mathbb{R}^{3}\right)
$$

and

$$
v\left(0,-t_{n}, \omega, v_{0, n}\right) \rightarrow v\left(0,-m, \omega, \tilde{v}_{m}\right) \quad \text { weakly in } \quad L^{2}\left(\mathbb{R}^{3}\right) .
$$

By (5.5) and (5.12)-(5.13) we find that

$$
\tilde{u}=u\left(0,-m, \omega, \tilde{u}_{m}\right) \quad \text { and } \quad \tilde{v}=v\left(0,-m, \omega, \tilde{v}_{m}\right) .
$$

Applying (4.27) to $\left(u\left(0,-m, \omega, \tilde{u}_{m}\right), v\left(0,-m, \omega, \tilde{v}_{m}\right)\right)$, by (5.14) we get

$$
E(\tilde{u}, \tilde{v})=e^{-4 \sigma m} E\left(\tilde{u}_{m}, \tilde{v}_{m}\right)+\int_{-m}^{0} e^{4 \sigma \xi} \Psi\left(u\left(\xi,-m, \omega, \tilde{u}_{m}\right), v\left(\xi,-m, \omega, \tilde{v}_{m}\right)\right) d \xi .
$$


Applying (4.27) to $\left(u\left(0,-m, \omega, u\left(-m,-t_{n}, \omega, u_{0, n}\right)\right), v\left(0,-m, \omega, v\left(-m,-t_{n}, \omega, v_{0, n}\right)\right)\right)$, by (5.11) and (4.25) we have

$$
\begin{gathered}
E\left(u\left(0,-t_{n}, \omega, u_{0, n}\right), v\left(0,-t_{n}, \omega, v_{0, n}\right)\right) \\
=e^{-4 \sigma m} E\left(u\left(-m,-t_{n}, \omega, u_{0, n}\right), v\left(-m,-t_{n}, \omega, v_{0, n}\right)\right) \\
+\int_{-m}^{0} e^{4 \sigma \xi} \Psi\left(u\left(\xi,-m, \omega, u\left(-m,-t_{n}, \omega, u_{0, n}\right)\right), v\left(\xi,-m, \omega, v\left(-m,-t_{n}, \omega, v_{0, n}\right)\right)\right) d \xi \\
=e^{-4 \sigma m} E\left(u\left(-m,-t_{n}, \omega, u_{0, n}\right), v\left(-m,-t_{n}, \omega, v_{0, n}\right)\right) \\
-2(\alpha-\delta-2 \sigma) \int_{-m}^{0} e^{4 \sigma \xi}\left\|v\left(\xi,-m, \omega, v\left(-m,-t_{n}, \omega, v_{0, n}\right)\right)\right\|^{2} d \xi \\
-2(\delta-2 \sigma)\left(\lambda+\delta^{2}-\alpha \delta\right) \int_{-m}^{0} e^{4 \sigma \xi}\left\|u\left(\xi,-m, \omega, u\left(-m,-t_{n}, \omega, u_{0, n}\right)\right)\right\|^{2} d \xi \\
-2(\delta-2 \sigma) \int_{-m}^{0} e^{4 \sigma \xi}\left\|\nabla u\left(\xi,-m, \omega, u\left(-m,-t_{n}, \omega, u_{0, n}\right)\right)\right\|^{2} d \xi \\
+8 \sigma \int_{-m}^{0} e^{4 \sigma \xi} \int_{\mathbb{R}^{3}} F\left(x, u\left(\xi,-m, \omega, u\left(-m,-t_{n}, \omega, u_{0, n}\right)\right)\right) d x d \xi \\
-2 \delta \int_{-m}^{0} e^{4 \sigma \xi} \int_{\mathbb{R}^{3}} u\left(\xi,-m, \omega, u\left(-m,-t_{n}, \omega, u_{0, n}\right)\right) \\
+2\left(\lambda+\delta^{2}-\alpha \delta\right) \int_{-m}^{0} e^{4 \sigma \xi} \int_{\mathbb{R}^{3}} h(x) u\left(\xi,-m, \omega, u\left(-m,-t_{n}, \omega, u_{0, n}\right)\right) \omega(\xi) d x d \xi \\
+2 \int_{-m}^{0} e^{4 \sigma \xi} \int_{\mathbb{R}^{3}} \nabla h(x) \cdot \nabla u\left(\xi,-m, \omega, u\left(-m,-t_{n}, \omega, u_{0, n}\right)\right) \omega(\xi) d x d \xi \\
+2 \int_{-m}^{0} e^{4 \sigma \xi} \int_{\mathbb{R}^{3}} h(x) f\left(x, u\left(\xi,-m, \omega, u\left(-m,-t_{n}, \omega, u_{0, n}\right)\right)\right) \omega(\xi) d x d \xi \\
+2 \int_{-m}^{0} e^{4 \sigma \xi} \int_{\mathbb{R}^{3}} g(x) v\left(\xi,-m, \omega, v\left(-m,-t_{n}, \omega, v_{0, n}\right)\right) d x d \xi \\
+2(\delta-\alpha) \int_{-m}^{0} e^{4 \sigma \xi} \int_{\mathbb{R}^{3}} h(x) v\left(\xi,-m, \omega, v\left(-m,-t_{n}, \omega, v_{0, n}\right)\right) \omega(\xi) d x d \xi \\
\left.\left.\left.(5.16)+t_{n}, \omega, u_{0, n}\right)\right)\right) d x d \xi
\end{gathered}
$$

Now, we need to deal with every term on the right-hand side of (5.16). For the first term, by (4.24) we have

$$
\begin{gathered}
e^{-4 \sigma m} E\left(u\left(-m,-t_{n}, \omega, u_{0, n}\right), v\left(-m,-t_{n}, \omega, v_{0, n}\right)\right) \\
=e^{-4 \sigma m}\left(\left\|v\left(-m,-t_{n}, \omega, v_{0, n}\right)\right\|^{2}+\left(\lambda+\delta^{2}-\alpha \delta\right)\left\|u\left(-m,-t_{n}, \omega, u_{0, n}\right)\right\|^{2}\right) \\
+e^{-4 \sigma m}\left(\left\|\nabla u\left(-m,-t_{n}, \omega, u_{0, n}\right)\right\|^{2}+2 \int_{\mathbb{R}^{3}} F\left(x, u\left(-m,-t_{n}, \omega, u_{0, n}\right)\right) d x\right),
\end{gathered}
$$

which along with (5.9) shows that for all $n \geq N_{4}$,

$$
\begin{aligned}
& e^{-4 \sigma m} E\left(u\left(-m,-t_{n}, \omega, u_{0, n}\right), v\left(-m,-t_{n}, \omega, v_{0, n}\right)\right) \\
\leq & c e^{-3 \sigma m} R(\omega)+2 e^{-4 \sigma m} \int_{\mathbb{R}^{3}} F\left(x, u\left(-m,-t_{n}, \omega, u_{0, n}\right)\right) d x .
\end{aligned}
$$


Using (3.8) to estimate the last term on the right-hand side of the above, since $\gamma \leq 3$ we get for all $n \geq N_{4}$,

$$
\begin{gathered}
\int_{\mathbb{R}^{3}} F\left(x, u\left(-m,-t_{n}, \omega, u_{0, n}\right)\right) d x \\
\leq c\left(\left\|u\left(-m,-t_{n}, \omega, u_{0, n}\right)\right\|^{2}+\left\|u\left(-m,-t_{n}, \omega, u_{0, n}\right)\right\|_{\gamma+1}^{\gamma+1}+1\right) \\
\leq c\left(\left\|u\left(-m,-t_{n}, \omega, u_{0, n}\right)\right\|^{2}+\left\|u\left(-m,-t_{n}, \omega, u_{0, n}\right)\right\|_{H^{1}}^{\gamma+1}+1\right),
\end{gathered}
$$

which along with (5.9) implies that for all $n \geq N_{4}$,

$$
\int_{\mathbb{R}^{3}} F\left(x, u\left(-m,-t_{n}, \omega, u_{0, n}\right)\right) d x \leq c\left(e^{\sigma m} R(\omega)+e^{2 \sigma m} R^{2}(\omega)+1\right) .
$$

By (5.17)-(5.18) we get that, for all $n \geq N_{4}$,

$$
e^{-4 \sigma m} E\left(u\left(-m,-t_{n}, \omega, u_{0, n}\right), v\left(-m,-t_{n}, \omega, v_{0, n}\right)\right) \leq c e^{-2 \sigma m}\left(1+R^{2}(\omega)\right) .
$$

Next, we deal with the second term on the right-hand side of (5.16). By (5.10) and Lemma 3.1 we find that for every $\xi \in[-m, 0]$, when $n \rightarrow \infty$,

$$
v\left(\xi,-m, \omega, v\left(-m,-t_{n}, \omega, v_{0, n}\right)\right) \rightarrow v\left(\xi,-m, \omega, \tilde{v}_{m}\right) \quad \text { in } \quad L^{2}\left(\mathbb{R}^{3}\right),
$$

which implies that, for all $\xi \in[-m, 0]$,

$$
\liminf _{n \rightarrow \infty}\left\|v\left(\xi,-m, \omega, v\left(-m,-t_{n}, \omega, v_{0, n}\right)\right)\right\|^{2} \geq\left\|v\left(\xi,-m, \omega, \tilde{v}_{m}\right)\right\|^{2} .
$$

By (5.20) and Fatou's lemma we obtain

$$
\begin{gathered}
\liminf _{n \rightarrow \infty} \int_{-m}^{0} e^{4 \sigma \xi}\left\|v\left(\xi,-m, \omega, v\left(-m,-t_{n}, \omega, v_{0, n}\right)\right)\right\|^{2} d \xi \\
\geq \int_{-m}^{0} e^{4 \sigma \xi} \liminf _{n \rightarrow \infty}\left\|v\left(\xi,-m, \omega, v\left(-m,-t_{n}, \omega, v_{0, n}\right)\right)\right\|^{2} d \xi \\
\geq \int_{-m}^{0} e^{4 \sigma \xi}\left\|v\left(\xi,-m, \omega, \tilde{v}_{m}\right)\right\|^{2} d \xi
\end{gathered}
$$

Therefore, by (4.2) we have

$$
\begin{gathered}
\limsup _{n \rightarrow \infty}-2(\alpha-\delta-2 \sigma) \int_{-m}^{0} e^{4 \sigma \xi}\left\|v\left(\xi,-m, \omega, v\left(-m,-t_{n}, \omega, v_{0, n}\right)\right)\right\|^{2} d \xi \\
=-2(\alpha-\delta-2 \sigma) \liminf _{n \rightarrow \infty} \int_{-m}^{0} e^{4 \sigma \xi}\left\|v\left(\xi,-m, \omega, v\left(-m,-t_{n}, \omega, v_{0, n}\right)\right)\right\|^{2} d \xi \\
\leq-2(\alpha-\delta-2 \sigma) \int_{-m}^{0} e^{4 \sigma \xi}\left\|v\left(\xi,-m, \omega, \tilde{v}_{m}\right)\right\|^{2} d \xi .
\end{gathered}
$$

Similarly, by (4.1), (4.2), (5.10) and Fatou's lemma, we can also prove that

$$
\begin{aligned}
& \limsup _{n \rightarrow \infty}-2(\delta-2 \sigma)\left(\lambda+\delta^{2}-\alpha \delta\right) \int_{-m}^{0} e^{4 \sigma \xi}\left\|u\left(\xi,-m, \omega, u\left(-m,-t_{n}, \omega, u_{0, n}\right)\right)\right\|^{2} d \xi \\
& (5.22) \leq-2(\delta-2 \sigma)\left(\lambda+\delta^{2}-\alpha \delta\right) \int_{-m}^{0} e^{4 \sigma \xi}\left\|u\left(\xi,-m, \omega, \tilde{u}_{m}\right)\right\|^{2} d \xi
\end{aligned}
$$


and

$$
\begin{gathered}
\limsup _{n \rightarrow \infty}-2(\delta-2 \sigma) \int_{-m}^{0} e^{4 \sigma \xi}\left\|\nabla u\left(\xi,-m, \omega, u\left(-m,-t_{n}, \omega, u_{0, n}\right)\right)\right\|^{2} d \xi \\
\leq-2(\delta-2 \sigma) \int_{-m}^{0} e^{4 \sigma \xi}\left\|\nabla u\left(\xi,-m, \omega, \tilde{u}_{m}\right)\right\|^{2} d \xi
\end{gathered}
$$

Next, we prove the convergence of the fifth term on the right-hand side of (5.16), which is a nonlinear term. We claim

$$
\begin{gathered}
\lim _{n \rightarrow \infty} \int_{-m}^{0} e^{4 \sigma \xi} \int_{\mathbb{R}^{3}} F\left(x, u\left(\xi,-m, \omega, u\left(-m,-t_{n}, \omega, u_{0, n}\right)\right)\right) d x d \xi \\
=\int_{-m}^{0} e^{4 \sigma \xi} \int_{\mathbb{R}^{3}} F\left(x, u\left(\xi,-m, \omega, \tilde{u}_{m}\right)\right) d x d \xi
\end{gathered}
$$

To prove (5.24) we write

$$
\begin{array}{r}
\left|\int_{-m}^{0} e^{4 \sigma \xi} \int_{\mathbb{R}^{3}}\left(F\left(x, u\left(\xi,-m, \omega, u\left(-m,-t_{n}, \omega, u_{0, n}\right)\right)\right)-F\left(x, u\left(\xi,-m, \omega, \tilde{u}_{m}\right)\right)\right) d x d \xi\right| \\
\leq \int_{-m}^{0} e^{4 \sigma \xi} \int_{|x|>k} \mid F\left(x, u\left(\xi,-m, \omega, u\left(-m,-t_{n}, \omega, u_{0, n}\right)\right)\right) \\
+\mid \int_{-m}^{0} e^{4 \sigma \xi} \int_{|x|<k} F\left(x, u\left(\xi,-m, \omega, u\left(-m,-t_{n}, \omega, u_{0, n}\right)\right)\right) \\
-F\left(x, u\left(\xi,-m, \omega, \tilde{u}_{m}\right)\right) d x d \xi \mid .
\end{array}
$$

Given $\epsilon>0$, by Lemma 4.2 we find that there are $k_{1}=k_{1}(\omega, \epsilon)>0$ and $N_{5}=$ $N_{5}(B, \omega, \epsilon) \geq N_{4}$ such that for all $k \geq k_{1}$ and $n \geq N_{5}$,

$$
\int_{|x|>k}\left|u\left(\xi,-t_{n}, \omega, u_{0, n}\right)\right|^{2} d x \leq \epsilon e^{-\sigma \xi},
$$

where $\xi \in\left[-t_{n}, 0\right]$. Hence, by (3.8) we obtain that for all $k \geq k_{1}$ and $n \geq N_{5}$,

$$
\begin{gathered}
\int_{|x|>k}\left|F\left(x, u\left(\xi,-t_{n}, \omega, u_{0, n}\right)\right)\right| d x \\
\leq \int_{|x|>k}\left(\left|u\left(\xi,-t_{n}, \omega, u_{0, n}\right)\right|^{2}+\left|u\left(\xi,-t_{n}, \omega, u_{0, n}\right)\right|^{\gamma+1}+\phi_{1}^{2}+\phi_{2}\right) d x \\
\leq \int_{|x|>k}\left(\phi_{1}^{2}+\phi_{2}\right) d x+\int_{|x|>k}\left|u\left(\xi,-t_{n}, \omega, u_{0, n}\right)\right|^{2} d x \\
\left.+\int_{|x|>k}\left|u\left(\xi,-t_{n}, \omega, u_{0, n}\right)\right|^{2 \gamma} d x\right)^{\frac{1}{2}}\left(\int_{|x|>k}\left|u\left(\xi,-t_{n}, \omega, u_{0, n}\right)\right|^{2} d x\right)^{\frac{1}{2}} \\
\leq \int_{|x|>k}\left(\phi_{1}^{2}+\phi_{2}\right) d x+\epsilon e^{-\sigma \xi}+\sqrt{\epsilon} e^{-\frac{\sigma}{2} \xi}\left(\int_{\mathbb{R}^{3}}\left|u\left(\xi,-t_{n}, \omega, u_{0, n}\right)\right|^{2 \gamma} d x\right)^{\frac{1}{2}} \\
\leq \int_{|x|>k}\left(\phi_{1}^{2}+\phi_{2}\right) d x+\epsilon e^{-\sigma \xi}+\sqrt{\epsilon} e^{-\frac{\sigma}{2} \xi}\left\|u\left(\xi,-t_{n}, \omega, u_{0, n}\right)\right\|_{H^{1}}^{\gamma},
\end{gathered}
$$


which along with the fact $\gamma \leq 3$ and (5.8) implies that

$$
\begin{aligned}
\int_{|x|>k} \mid F(x, & \left.u\left(\xi,-t_{n}, \omega, u_{0, n}\right)\right) \mid d x \\
& \leq \int_{|x|>k}\left(\phi_{1}^{2}+\phi_{2}\right) d x+\epsilon e^{-\sigma \xi}+c \sqrt{\epsilon} e^{-\frac{\sigma}{2} \xi}\left(1+e^{-\frac{3 \sigma}{2} \xi} R^{\frac{3}{2}}(\omega)\right) .
\end{aligned}
$$

Notice that there is $k_{2}=k_{2}(\epsilon)>0$ such that for all $k \geq k_{2}$, the first term on the right-hand side of (5.27) is bounded by $\epsilon$. Therefore, for all $\xi \leq 0, n \geq N_{5}$ and $k \geq k_{3}=\max \left\{k_{1}, k_{2}\right\}$,

$$
\int_{|x|>k}\left|F\left(x, u\left(\xi,-t_{n}, \omega, u_{0, n}\right)\right)\right| d x \leq \epsilon+e^{-2 \sigma \xi}\left(\epsilon+\sqrt{\epsilon} c+\sqrt{\epsilon} c R^{\frac{3}{2}}(\omega)\right) .
$$

On the other hand, there exists $k_{4}=k_{4}(m, \omega, \epsilon) \geq k_{3}$ such that for all $k \geq k_{4}$,

$$
\int_{-m}^{0} e^{4 \sigma \xi} \int_{|x|>k}\left|F\left(x, u\left(\xi,-m, \omega, \tilde{u}_{m}\right)\right)\right| d x d \xi \leq \epsilon
$$

By (5.28)-(5.29), the first term on the right-hand side of (5.25) satisfies, for all $n \geq N_{5}$ and $k \geq k_{4}$,

$$
\begin{gathered}
\int_{-m}^{0} e^{4 \sigma \xi} \int_{|x|>k}\left|F\left(x, u\left(\xi,-m, \omega, u\left(-m,-t_{n}, \omega, u_{0, n}\right)\right)\right)-F\left(x, u\left(\xi,-m, \omega, \tilde{u}_{m}\right)\right)\right| d x d \xi \\
=\int_{-m}^{0} e^{4 \sigma \xi} \int_{|x|>k}\left|F\left(x, u\left(\xi,-t_{n}, \omega, u_{0, n}\right)\right)-F\left(x, u\left(\xi,-m, \omega, \tilde{u}_{m}\right)\right)\right| d x d \xi \\
\leq \int_{-m}^{0} e^{4 \sigma \xi} \int_{|x|>k}\left|F\left(x, u\left(\xi,-t_{n}, \omega, u_{0, n}\right)\right)\right| d x d \xi \\
\quad+\int_{-m}^{0} e^{4 \sigma \xi} \int_{|x|>k}\left|F\left(x, u\left(\xi,-m, \omega, \tilde{u}_{m}\right)\right)\right| d x d \xi \\
\leq \epsilon+\int_{-m}^{0} e^{4 \sigma \xi} d \xi+\left(\epsilon+\sqrt{\epsilon} c+\sqrt{\epsilon} c R^{\frac{3}{2}}(\omega)\right) \int_{-m}^{0} e^{2 \sigma \xi} d \xi \\
\leq \sqrt{\epsilon} c\left(1+R^{\frac{3}{2}}(\omega)\right) \quad \text { for all } \epsilon \leq 1 .
\end{gathered}
$$

To deal with the second term on the right-hand side of (5.25), we notice that, by (5.10) and Lemma 3.1, when $n \rightarrow \infty$,

$$
u\left(\xi,-m, \omega, u\left(-m,-t_{n}, \omega, u_{0, n}\right)\right) \rightarrow u\left(\xi,-m, \omega, \tilde{u}_{m}\right) \quad \text { weakly in } H^{1}\left(\mathbb{R}^{3}\right)
$$

for $\xi \in[-m, 0]$. By (5.31) and the compactness of embedding $H^{1}\left(Q_{k}\right) \hookrightarrow L^{2}\left(Q_{k}\right)$, we find that, for $\xi \in[-m, 0]$,

$$
u\left(\xi,-m, \omega, u\left(-m,-t_{n}, \omega, u_{0, n}\right)\right) \rightarrow u\left(\xi,-m, \omega, \tilde{u}_{m}\right) \quad \text { strongly in } L^{2}\left(Q_{k}\right)
$$


We also have

$$
\begin{aligned}
& \text { (5.33) }\left|\int_{|x|<k}\left(F\left(x, u\left(\xi,-m, \omega, u\left(-m,-t_{n}, \omega, u_{0, n}\right)\right)\right)-F\left(x, u\left(\xi,-m, \omega, \tilde{u}_{m}\right)\right)\right) d x\right| \\
& =\left|\int_{|x|<k} \frac{\partial F}{\partial u}(x, \bar{u})\left(u\left(\xi,-m, \omega, u\left(-m,-t_{n}, \omega, u_{0, n}\right)\right)-u\left(\xi,-m, \omega, \tilde{u}_{m}\right)\right) d x\right| \\
& \quad=\left|\int_{|x|<k} f(x, \bar{u})\left(u\left(\xi,-m, \omega, u\left(-m,-t_{n}, \omega, u_{0, n}\right)\right)-u\left(\xi,-m, \omega, \tilde{u}_{m}\right)\right) d x\right| \\
& \left.\leq\left(\int_{\mathbb{R}^{3}}|f(x, \bar{u})|^{2} d x\right)^{\frac{1}{2}} \| u\left(\xi,-m, \omega, u\left(-m,-t_{n}, \omega, u_{0, n}\right)\right)-u\left(\xi,-m, \omega, \tilde{u}_{m}\right)\right) \|_{L^{2}\left(Q_{k}\right)} .
\end{aligned}
$$

By (3.4) and (5.8) we get

$$
\begin{aligned}
& \left(\int_{\mathbb{R}^{3}}|f(x, \bar{u})|^{2} d x\right)^{\frac{1}{2}} \\
& \quad \leq c\left(\left\|u\left(\xi,-m, \omega, u\left(-m,-t_{n}, \omega, u_{0, n}\right)\right)\right\|_{H^{1}}^{\gamma}+\left\|u\left(\xi,-m, \omega, \tilde{u}_{m}\right)\right\|^{\gamma}+\left\|\phi_{1}\right\|^{2}\right) \\
& \quad \leq c\left(\left\|u\left(\xi,-t_{n}, \omega, u_{0, n}\right)\right\|_{H^{1}}^{\gamma}+\left\|u\left(\xi,-m, \omega, \tilde{u}_{m}\right)\right\|^{\gamma}+\left\|\phi_{1}\right\|^{2}\right) \\
& \quad \leq c\left(e^{-\frac{\sigma \gamma}{2} \xi} R^{\frac{\gamma}{2}}(\omega)+\left\|u\left(\xi,-m, \omega, \tilde{u}_{m}\right)\right\|^{\gamma}+\left\|\phi_{1}\right\|^{2}\right),
\end{aligned}
$$

which along with (5.32) and (5.33) implies that, as $n \rightarrow \infty$,

$$
\int_{|x|<k} F\left(x, u\left(\xi,-m, \omega, u\left(-m,-t_{n}, \omega, u_{0, n}\right)\right)\right) d x \rightarrow \int_{|x|<k} F\left(x, u\left(\xi,-m, \omega, \tilde{u}_{m}\right)\right) d x .
$$

It follows from (5.8), (5.35) and the dominated convergence theorem that, when $n \rightarrow \infty$,

$$
\begin{gathered}
\int_{-m}^{0} e^{4 \sigma \xi} \int_{|x|<k} F\left(x, u\left(\xi,-m, \omega, u\left(-m,-t_{n}, \omega, u_{0, n}\right)\right)\right) d x d \xi \\
\rightarrow \int_{-m}^{0} e^{4 \sigma \xi} \int_{|x|<k} F\left(x, u\left(\xi,-m, \omega, \tilde{u}_{m}\right)\right) d x d \xi .
\end{gathered}
$$

Therefore, there exists $N_{6} \geq N_{5}$ such that for all $n \geq N_{6}$,

$$
\begin{array}{r}
\mid \int_{-m}^{0} e^{4 \sigma \xi} \int_{|x|<k}\left(F\left(x, u\left(\xi,-m, \omega, u\left(-m,-t_{n}, \omega, u_{0, n}\right)\right)\right)\right. \\
\left.-F\left(x, u\left(\xi,-m, \omega, \tilde{u}_{m}\right)\right)\right) d x d \xi \mid \leq \epsilon,
\end{array}
$$

which along with (5.25) and (5.30) implies (5.24). By an argument similar to the proof of (5.24), we can also show the convergence of the sixth term on the right-hand side of (5.16) (details are omitted). That is, we have that, as $n \rightarrow \infty$,

$$
\begin{aligned}
\int_{-m}^{0} e^{4 \sigma \xi} \int_{\mathbb{R}^{3}} u\left(\xi,-m, \omega, u\left(-m,-t_{n}, \omega, u_{0, n}\right)\right) \\
\quad \times f\left(x, u\left(\xi,-m, \omega, u\left(-m,-t_{n}, \omega, u_{0, n}\right)\right)\right) d x d \xi \\
\rightarrow \int_{-m}^{0} e^{4 \sigma \xi} \int_{\mathbb{R}^{3}} u\left(\xi,-m, \omega, \tilde{u}_{m}\right) \times f\left(x, u\left(\xi,-m, \omega, \tilde{u}_{m}\right)\right) d x d \xi
\end{aligned}
$$


The convergence of the remaining terms on the right-hand side of (5.16) is given below, which can be proved by a similar (actually simpler) procedure.

$$
\begin{gathered}
\int_{-m}^{0} e^{4 \sigma \xi} \int_{\mathbb{R}^{3}} h(x) u\left(\xi,-m, \omega, u\left(-m,-t_{n}, \omega, u_{0, n}\right)\right) \omega(\xi) d x d \xi \\
\rightarrow \int_{-m}^{0} e^{4 \sigma \xi} \int_{\mathbb{R}^{3}} h(x) u\left(\xi,-m, \omega, \tilde{u}_{m}\right) \omega(\xi) d x d \xi
\end{gathered}
$$

$$
\int_{-m}^{0} e^{4 \sigma \xi} \int_{\mathbb{R}^{3}} \nabla h(x) \cdot \nabla u\left(\xi,-m, \omega, u\left(-m,-t_{n}, \omega, u_{0, n}\right)\right) \omega(\xi) d x d \xi
$$

$$
\begin{gathered}
\rightarrow \int_{-m}^{0} e^{4 \sigma \xi} \int_{\mathbb{R}^{3}} \nabla h(x) \cdot \nabla u\left(\xi,-m, \omega, \tilde{u}_{m}\right) \omega(\xi) d x d \xi . \\
\int_{-m}^{0} e^{4 \sigma \xi} \int_{\mathbb{R}^{3}} h(x) f\left(x, u\left(\xi,-m, \omega, u\left(-m,-t_{n}, \omega, u_{0, n}\right)\right)\right) \omega(\xi) d x d \xi \\
\rightarrow \int_{-m}^{0} e^{4 \sigma \xi} \int_{\mathbb{R}^{3}} h(x) f\left(x, u\left(\xi,-m, \omega, \tilde{u}_{m}\right)\right) \omega(\xi) d x d \xi . \\
\int_{-m}^{0} e^{4 \sigma \xi} \int_{\mathbb{R}^{3}} g(x) v\left(\xi,-m, \omega, v\left(-m,-t_{n}, \omega, v_{0, n}\right)\right) d x d \xi \\
\quad \rightarrow \int_{-m}^{0} e^{4 \sigma \xi} \int_{\mathbb{R}^{3}} g(x) v\left(\xi,-m, \omega, \tilde{v}_{m}\right) d x d \xi . \\
\int_{-m}^{0} e^{4 \sigma \xi} \int_{\mathbb{R}^{3}} h(x) v\left(\xi,-m, \omega, v\left(-m,-t_{n}, \omega, v_{0, n}\right)\right) \omega(\xi) d x d \xi \\
\rightarrow \int_{-m}^{0} e^{4 \sigma \xi} \int_{\mathbb{R}^{3}} h(x) v\left(\xi,-m, \omega, \tilde{v}_{m}\right) \omega(\xi) d x d \xi .
\end{gathered}
$$

Now, taking the limit of (5.16) as $n \rightarrow \infty$, by (5.19), (5.21) $-(\sqrt{5.24})$ and (5.37)-(5.42) we find that

$$
\begin{gathered}
\limsup _{n \rightarrow \infty} E\left(u\left(0,-t_{n}, \omega, u_{0, n}\right), v\left(0,-t_{n}, \omega, v_{0, n}\right)\right) \\
\leq c e^{-2 \sigma m}\left(1+R^{2}(\omega)\right)-2(\alpha-\delta-2 \sigma) \int_{-m}^{0} e^{4 \sigma \xi}\left\|v\left(\xi,-m, \omega, \tilde{v}_{m}\right)\right\|^{2} d \xi \\
-2(\delta-2 \sigma)\left(\lambda+\delta^{2}-\alpha \delta\right) \int_{-m}^{0} e^{4 \sigma \xi}\left\|u\left(\xi,-m, \omega, \tilde{u}_{m}\right)\right\|^{2} d \xi \\
-2(\delta-2 \sigma) \int_{-m}^{0} e^{4 \sigma \xi}\left\|\nabla u\left(\xi,-m, \omega, \tilde{u}_{m}\right)\right\|^{2} d \xi \\
+8 \sigma \int_{-m}^{0} e^{4 \sigma \xi} \int_{\mathbb{R}^{3}} F\left(x, u\left(\xi,-m, \omega, \tilde{u}_{m}\right)\right) d x d \xi \\
-2 \delta \int_{-m}^{0} e^{4 \sigma \xi} \int_{\mathbb{R}^{3}} u\left(\xi,-m, \omega, \tilde{u}_{m}\right) \times f\left(x, u\left(\xi,-m, \omega, \tilde{u}_{m}\right)\right) d x d \xi \\
+2\left(\lambda+\delta^{2}-\alpha \delta\right) \int_{-m}^{0} e^{4 \sigma \xi} \int_{\mathbb{R}^{3}} h(x) u\left(\xi,-m, \omega, \tilde{u}_{m}\right) \omega(\xi) d x d \xi \\
+2 \int_{-m}^{0} e^{4 \sigma \xi} \int_{\mathbb{R}^{3}} \nabla h(x) \cdot \nabla u\left(\xi,-m, \omega, \tilde{u}_{m}\right) \omega(\xi) d x d \xi
\end{gathered}
$$




$$
\begin{aligned}
& +2 \int_{-m}^{0} e^{4 \sigma \xi} \int_{\mathbb{R}^{3}} h(x) f\left(x, u\left(\xi,-m, \omega, \tilde{u}_{m}\right)\right) \omega(\xi) d x d \xi \\
& \quad+2 \int_{-m}^{0} e^{4 \sigma \xi} \int_{\mathbb{R}^{3}} g(x) v\left(\xi,-m, \omega, \tilde{v}_{m}\right) d x d \xi \\
& +2(\delta-\alpha) \int_{-m}^{0} e^{4 \sigma \xi} \int_{\mathbb{R}^{3}} h(x) v\left(\xi,-m, \omega, \tilde{v}_{m}\right) \omega(\xi) d x d \xi .
\end{aligned}
$$

It follows from (4.25) and (5.43) that

$$
\begin{gathered}
\limsup _{n \rightarrow \infty} E\left(u\left(0,-t_{n}, \omega, u_{0, n}\right), v\left(0,-t_{n}, \omega, v_{0, n}\right)\right) \\
\leq c e^{-2 \sigma m}\left(1+R^{2}(\omega)\right)+\int_{-m}^{0} e^{4 \sigma \xi} \Psi\left(u\left(\xi,-m, \omega, \tilde{u}_{m}\right), v\left(\xi,-m, \omega, \tilde{v}_{m}\right)\right) d \xi .
\end{gathered}
$$

By (5.15) and (5.44) we find that

$$
\begin{aligned}
& \limsup _{n \rightarrow \infty} E\left(u\left(0,-t_{n}, \omega, u_{0, n}\right), v\left(0,-t_{n}, \omega, v_{0, n}\right)\right) \\
\leq & c e^{-2 \sigma m}\left(1+R^{2}(\omega)\right)-e^{-4 \sigma m} E\left(\tilde{u}_{m}, \tilde{v}_{m}\right)+E(\tilde{u}, \tilde{v}) .
\end{aligned}
$$

For the second term on the right-hand side of (5.45), by (4.24) and (3.6) we have

$$
-e^{-4 \sigma m} E\left(\tilde{u}_{m}, \tilde{v}_{m}\right) \leq 2 e^{-4 \sigma m} \int_{\mathbb{R}^{3}} \phi_{3}(x) d x .
$$

It follows from (5.45)-(5.46) that

$$
\begin{gathered}
\limsup _{n \rightarrow \infty} E\left(u\left(0,-t_{n}, \omega, u_{0, n}\right), v\left(0,-t_{n}, \omega, v_{0, n}\right)\right) \\
\leq c e^{-2 \sigma m}\left(1+R^{2}(\omega)\right)+2 e^{-4 \sigma m} \int_{\mathbb{R}^{3}} \phi_{3}(x) d x+E(\tilde{u}, \tilde{v}) .
\end{gathered}
$$

Let $m \rightarrow \infty$. Then we get that

$$
\limsup _{n \rightarrow \infty} E\left(u\left(0,-t_{n}, \omega, u_{0, n}\right), v\left(0,-t_{n}, \omega, v_{0, n}\right)\right) \leq E(\tilde{u}, \tilde{v}) .
$$

On the other hand, it follows from (5.28) and (5.35) with $\xi=0$ that, as $n \rightarrow \infty$,

$$
\int_{\mathbb{R}^{3}} F\left(x, u\left(0,-t_{n}, \omega, u_{0, n}\right)\right) d x \rightarrow \int_{\mathbb{R}^{3}} F(x, \tilde{u}) d x,
$$

which along with (4.24) shows that

$$
\begin{aligned}
& \limsup _{n \rightarrow \infty} E\left(u\left(0,-t_{n}, \omega, u_{0, n}\right), v\left(0,-t_{n}, \omega, v_{0, n}\right)\right)=2 \int_{\mathbb{R}^{3}} F(x, \tilde{u}) d x \\
&+\limsup _{n \rightarrow \infty}\left(\left\|v\left(0,-t_{n}, \omega, v_{0, n}\right)\right\|^{2}+\left(\lambda+\delta^{2}-\alpha \delta\right)\left\|u\left(0,-t_{n}, \omega, u_{0, n}\right)\right\|^{2}\right. \\
&\left.+\left\|\nabla u\left(0,-t_{n}, \omega, u_{0, n}\right)\right\|^{2}\right) .
\end{aligned}
$$

Substituting the above equality into (5.48), by (4.24) we obtain that

$$
\begin{aligned}
\limsup _{n \rightarrow \infty}\left(\left\|v\left(0,-t_{n}, \omega, v_{0, n}\right)\right\|^{2}+(\lambda\right. & \left.+\delta^{2}-\alpha \delta\right)\left\|u\left(0,-t_{n}, \omega, u_{0, n}\right)\right\|^{2} \\
& \left.+\left\|\nabla u\left(0,-t_{n}, \omega, u_{0, n}\right)\right\|^{2}\right) \\
\leq\|\tilde{v}\|^{2}+ & \left(\lambda+\delta^{2}-\alpha \delta\right)\|\tilde{u}\|^{2}+\|\nabla \tilde{u}\|^{2} .
\end{aligned}
$$


Notice that the left and right expressions are equivalent norms of $H^{1}\left(\mathbb{R}^{3}\right) \times L^{2}\left(\mathbb{R}^{3}\right)$. Therefore, by (4.1) and (5.49) we find that

$$
\limsup _{n \rightarrow \infty}\left(\left\|u\left(0,-t_{n}, \omega, u_{0, n}\right)\right\|_{H^{1}}^{2}+\left\|v\left(0,-t_{n}, \omega, v_{0, n}\right)\right\|^{2}\right) \leq\|\tilde{u}\|_{H^{1}}^{2}+\|\tilde{v}\|^{2},
$$

which implies (5.7). Finally, we get the following strong convergence by (5.5)-(5.7):

$$
\left(u\left(0,-t_{n}, \omega, u_{0, n}\right), v\left(0,-t_{n}, \omega, v_{0, n}\right)\right) \rightarrow(\tilde{u}, \tilde{v}) \text { strongly in } H^{1}\left(\mathbb{R}^{3}\right) \times L^{2}\left(\mathbb{R}^{3}\right) .
$$

This completes the proof.

As an immediate consequence of Lemma 5.1 we see that the random dynamical system $\Phi$ is pullback asymptotically compact in $H^{1}\left(\mathbb{R}^{3}\right) \times L^{2}\left(\mathbb{R}^{3}\right)$.

Lemma 5.2. Assume that $g \in L^{2}\left(\mathbb{R}^{3}\right), h \in H^{1}\left(\mathbb{R}^{3}\right)$ and (3.4)-(3.7) hold. Then the random dynamical system $\Phi$ is $\mathcal{D}$-pullback asymptotically compact in $H^{1}\left(\mathbb{R}^{3}\right) \times$ $L^{2}\left(\mathbb{R}^{3}\right)$; that is, for P-a.e. $\omega \in \Omega$, the sequence $\left\{\Phi\left(t_{n}, \theta_{-t_{n}} \omega,\left(u_{0, n}, z_{0, n}\right)\right)\right\}$ has a convergent subsequence in $H^{1}\left(\mathbb{R}^{3}\right) \times L^{2}\left(\mathbb{R}^{3}\right)$ provided $t_{n} \rightarrow \infty$ and $\left(u_{0, n}, z_{0, n}\right) \in$ $B\left(\theta_{-t_{n}} \omega\right)$ with $B=\{B(\omega)\}_{\omega \in \Omega} \in \mathcal{D}$.

We are now in a position to prove the existence of a random attractor for the stochastic wave equation.

Theorem 5.3. Assume that $g \in L^{2}\left(\mathbb{R}^{3}\right), h \in H^{1}\left(\mathbb{R}^{3}\right)$ and (3.4)-(3.7) hold. Then the random dynamical system $\Phi$ has a unique $\mathcal{D}$-random attractor $\{\mathcal{A}(\omega)\}_{\omega \in \Omega}$ in $H^{1}\left(\mathbb{R}^{3}\right) \times L^{2}\left(\mathbb{R}^{3}\right)$.

Proof. Notice that $\Phi$ has a closed absorbing set $\tilde{B}=\{\tilde{B}(\omega)\}_{\omega \in \Omega}$ in $\mathcal{D}$ by (5.2)(5.3), and is $\mathcal{D}$-pullback asymptotically compact in $H^{1}\left(\mathbb{R}^{3}\right) \times L^{2}\left(\mathbb{R}^{3}\right)$ by Lemma 5.2. Hence the existence of a unique $\mathcal{D}$-random attractor immediately follows from Proposition 2.9.

\section{REFERENCES}

[1] L. Arnold, Random Dynamical Systems, Springer-Verlag, 1998. MR1723992 (2000m:37087)

[2] J.M. Arrieta, A.N. Carvalho and J.K. Hale, A damped hyperbolic equation with critical exponent, Communications in Partial Differential Equations, 17 (1992), 841-866. MR.1177295 (93f:35145)

[3] A.V. Babin and M.I. Vishik, Attractors of Evolution Equations, North-Holland, Amsterdam, 1992. MR1156492 (93d:58090)

[4] J.M. Ball, Global attractors for damped semilinear wave equations, Discrete Continuous Dynamical Systems, 10 (2004), 31-52. MR2026182 (2005a:37149)

[5] J.M. Ball, Continuity properties and global attractors of generalized semiflows and the NavierStokes equations, J. Nonlinear Sci., 7 (1997), 475-502. MR.1462276 (98j:58071a)

[6] P.W. Bates, H. Lisei and K. Lu, Attractors for stochastic lattice dynamical systems, Stoch. Dyn., 6 (2006), 1-21. MR2210679 (2006m:60077)

[7] P.W. Bates, K. Lu and B. Wang, Random attractors for stochastic reaction-diffusion equations on unbounded domains, J. Differential Equations, 246 (2009), 845-869. MR2468738 (2009i:35357)

[8] Z. Brzezniak and Y. Li, Asymptotic compactness and absorbing sets for 2d stochastic NavierStokes equations on some unbounded domains, Transactions of American Math. Soc., 358 (2006), 5587-5629. MR2238928(2008i:60101)

[9] T. Caraballo, J. A. Langa and J. C. Robinson, A stochastic pitchfork bifurcation in a reactiondiffusion equation, Proc. R. Soc. Lond. A, 457 (2001), 2041-2061. MR1857922(2003a:60106)

[10] T. Caraballo, J. Real, I.D. Chueshov, Pullback attractors for stochastic heat equations in materials with memory, Discrete Continuous Dynamical Systems B, 9 (2008), 525-539. MR2379425 (2008m:60111) 
[11] I. Chueshov and I. Lasiecka, Attractors for second-order evolution equations with a nonlinear damping, J. Dynam. Differential Equations, 16 (2004), 469-512. MR.2105786 (2005g:37149)

[12] I. Chueshov and M. Scheutzow, On the structure of attractors and invariant measures for a class of monotone random systems, Dynamical Systems, 19 (2004), 127-144. MR2060422 (2005c:37095)

[13] H. Crauel, A. Debussche and F. Flandoli, Random attractors, J. Dyn. Diff. Eqns., 9 (1997), 307-341. MR:1451294 (98c:60066)

[14] H. Crauel and F. Flandoli, Attractors for random dynamical systems, Probab. Theory Related Fields, 100 (1994), 365-393. MR.1305587 (95k:58092)

[15] G. Da Prato, An Introduction to Infinite-Dimensional Analysis, Springer-Verlag, Berlin, 2006. MR2244975 (2009a:46001)

[16] E. Feireisl, Attractors for semilinear damped wave equations on $\mathbb{R}^{3}$, Nonlinear Analysis TMA, 23 (1994), 187-195. MR 1289126 (95i:35188)

[17] E. Feireisl, Global attractors for semilinear damped wave equations with supercritical exponent, J. Differential Equations, 116 (1995) 431-447. MR.1318582 (96a:35120)

[18] E. Feireisl and E. Zuazua, Global attractors for semilinear wave equations with locally distributed nonlinear damping and critical exponent, Communications in Partial Differential Equations 18 (1993) 1539-1555. MR:1239923 (95c:35172)

[19] F. Flandoli and B. Schmalfu $\beta$, Random attractors for the 3D stochastic Navier-Stokes equation with multiplicative noise, Stoch. Stoch. Rep., 59 (1996), 21-45. MR.1427258 (98g:60113)

[20] J.K. Hale, Asymptotic Behavior of Dissipative Systems, American Mathematical Society, Providence, RI, 1988. MR941371 (89g:58059)

[21] A. Haraux, Semi-Linear Hyperbolic Problems in Bounded Domains, Mathematical Reports, Vol. 3, Part 1, Harwood Academic Publishers, New York, 1987. MR1078761 (91m:35150)

[22] A. Kh. Khanmamedov, Global attractors for wave equations with nonlinear interior damping and critical exponents, J. Differential Equations, 230 (2006), 702-719. MR2269940 (2007i:37150)

[23] P.E. Kloeden and J.A. Langa, Flattening, squeezing and the existence of random attractors, Proc. Royal Soc. London Series A., 463 (2007), 163-181. MR2281716 (2008a:37056)

[24] N. Ju, The $H^{1}$-compact global attractor for the solutions to the Navier-Stokes equations in two-dimensional unbounded domains, Nonlinearity, 13 (2000), 1227-1238. MR 1767956 (2001f:37131)

[25] I. Moise, R. Rosa and X. Wang, Attractors for non-compact semigroups via energy equations, Nonlinearity, 11 (1998), 1369-1393. MR.1644413 (99h:35020)

[26] I. Moise, R. Rosa and X. Wang, Attractors for non-compact nonautonomous systems via energy equations, Discrete Continuous Dynamical Systems, 10 (2004), 473-496. MR2026206 (2004m:37151)

[27] M. Prizzi and K.P. Rybakowski, Attractors for semilinear damped wave equations on arbitrary unbounded domains, Topol. Methods Nonlinear Anal., 31 (2008),49-82. MR 2420655 (2009h:35287)

[28] M. Prizzi and K. P. Rybakowski, Attractors for singularly perturbed damped wave equations on unbounded domains, Topol. Methods Nonlinear Anal., 32 (2008), 1-20. MR2466799

[29] M. Prizzi, Regularity of invariant sets in semilinear damped wave equations, J. Differential Equations, 247 (2009), 3315-3337. MR2571579 (2010k:35317)

[30] R. Sell and Y. You, Dynamics of Evolutionary Equations, Springer-Verlag, New York, 2002. MR.1873467 (2003f:37001b)

[31] W.A. Strauss, Nonlinear Wave Equations, CBMS Regional Conference Series in Mathematics, Vol. 73, American Mathematical Society, 1990. MR.1777631 (2001c:35002)

[32] C. Sun, D. Cao and J. Duan, Non-autonomous dynamics of wave equations with nonlinear damping and critical nonlinearity, Nonlinearity, 19 (2006), 2645-2665. MR 2267722 (2007h:35235)

[33] C. Sun, M. Yang and C. Zhong, Global attractors for the wave equation with nonlinear damping, J. Differential Equations, 227 (2006), 427-443. MR2237675 (2007j:37140)

[34] R. Temam, Infinite-Dimensional Dynamical Systems in Mechanics and Physics, SpringerVerlag, New York, 1997. MR1441312 (98b:58056)

[35] B. Wang, Attractors for reaction-diffusion equations in unbounded domains, Physica D, 128 (1999), 41-52. MR 1685247 (2000a:35126) 
[36] B. Wang, Random Attractors for the Stochastic Benjamin-Bona-Mahony Equation on Unbounded Domains, J. Differential Equations, 246 (2009), 2506-2537. MR2498851

[37] B. Wang, Random attractors for the stochastic FitzHugh-Nagumo system on unbounded domains, Nonlinear Analysis, TMA, 71 (2009), 2811-2828. MR2532807

[38] B. Wang and X. Gao, Random attractors for wave equations on unbounded domains, Discrete Continuous Dynamical Systems, special (2009), 800-809. MR.2648205

[39] B. Wang, Upper semicontinuity of random attractors for non-compact random dynamical systems, Electronic Journal of Differential Equations, 2009 (2009), No. 139, 1-18. MR2558811

[40] X. Wang, An energy equation for the weakly damped driven nonlinear Schrödinger equations and its applications, Physica D, 88 (1995), 167-175. MR1360882 (96h:35215)

[41] S. Zhou, F. Yin and Z. Ouyang, Random attractor for damped nonlinear wave equations with white noise, SIAM J. Appl. Dyn. Syst., 4 (2005), 883-903. MR2179491(2006g:60096)

Department of Mathematics, New Mexico Institute of Mining and Technology, Socorro, New Mexico 87801

E-mail address: bwang@nmt.edu 